Working Paper 14-24

Statistics and Econometrics Series (16)

July 2014
Departamento de Estadística Universidad Carlos III de Madrid

Calle Madrid, 126

28903 Getafe (Spain)

Fax (34) 91 624-98-48

\title{
DISENTANGLED JUMP-ROBUST REALIZED COVARIANCES AND CORRELATIONS WITH NON-SYNCHRONOUS PRICES
}

\author{
Harry Vander Elst ${ }^{1}$ and David Veredas ${ }^{2,3}$
}

\begin{abstract}
We study the class of disentangled realized estimators for the integrated covariance matrix of Brownian semimartingales with finite activity jumps. These estimators separate correlations and volatilities. We analyse - in a through Monte Carlo study different combinations of quantile-and-median-based realized volatilities, and four estimators of realized correlations with three synchronization schemes. Their finite sample properties are studied under four data generating processes and in presence, or not, of microstructure noise, and under synchronous and asynchronous trading. The main finding is that pre-averaged disentangled estimators provide a precise, computationally efficient and easy alternative to measure integrated covariances on basis of noisy and asynchronous prices. Moreover, the gain is not only statistical but also financial. A minimum variance portfolio application shows the superiority of the disentangled realized estimators in terms of numerous performance metrics.
\end{abstract}

Keywords: Realized measures, noise, jumps, synchronization.

\footnotetext{
${ }^{1}$ Corresponding autor. ECARES, Solvay Brussels School of Economics and Management, Université libre de Bruxelles 50, Av Roosevelt CP114, B1050 Brussels, Belgium. Tel: +32 (0)26504502; Fax: +3226504475; havdelst@ulb.ac.be

${ }^{2}$ ECARES, Solvay Brussels School of Economics and Management, Université libre de Bruxelles.

${ }^{3}$ Institute of Information Theory and Automation
} 


\title{
Disentangled Jump-Robust ReAlized Covariances And Correlations With NON-SYNCHRONOUS PRICES
}

\author{
Harry Vander Elst ${ }^{1}$ and David Veredas ${ }^{2,3}$
}

First draft: July 2014

\begin{abstract}
We study the class of disentangled realized estimators for the integrated covariance matrix of Brownian semimartingales with finite activity jumps. These estimators separate correlations and volatilities. We analyze - in a thorough Monte Carlo study - different combinations of quantile-and-median-based realized volatilities, and four estimators of realized correlations with three synchronization schemes. Their finite sample properties are studied under four data generating processes and in presence, or not, of microstructure noise, and under synchronous and asynchronous trading. The main finding is that pre-averaged disentangled estimators provide a precise, computationally efficient and easy alternative to measure integrated covariances on basis of noisy and asynchronous prices. Moreover, the gain is not only statistical but also financial. A minimum variance portfolio application shows the superiority of the disentangled realized estimators in terms of numerous performance metrics.
\end{abstract}

Keywords: Realized measures, noise, jumps, synchronization.

JEL Classification: C58

\footnotetext{
${ }^{1}$ Corresponding author. ECARES, Solvay Brussels School of Economics and Management, Université libre de Bruxelles.. 50, Av Roosevelt CP114, B1050 Brussels, Belgium. Tel: +32(0)26504502; Fax: +3226504475; havdelst@ulb.ac.be

${ }^{2}$ ECARES, Solvay Brussels School of Economics and Management, Université libre de Bruxelles.

${ }^{3}$ Institute of Information Theory and Automation, Academy of Sciences of the Czech Republic.
} 


\section{Introduction}

Most of the activities in financial institutions such as risk management, portfolio selection and asset pricing require precise measures that summarize the relationships between risk factors. Among these parameters, correlations and volatilities are of paramount importance as they provide, on the one hand, insights on the links between assets and, on the other hand, necessary inputs for various risk measures.

Realized correlations and volatilities are nonparametric estimators of the ex-post variation of prices. In both the univariate and multivariate cases, the baseline estimators (simply obtained by summing intraday squared returns or intraday product of returns) face numerous drawbacks.

First, empirical properties of asset prices suggest the existence of jumps. Jumps enable to accommodate fat tails in the empirical density of returns and smiles in volatility surfaces of option prices. Models for jumps can be of two types: either with finite amount of large jumps or with infinite amount of small jumps. Jumps introduce an additional source of variation in prices which is of interest for many purposes, but lead to biases in covariance measurements. Several estimators are able to separate these sources of variations. For the univariate case, see e.g. bipower variation (Barndorff-Nielsen and Shephard (2004b)), quantile-based realized variances (Christensen et al. (2010b)), and MinRV and MedRV (Andersen et al. (2012)). In the multivariate case, bipower covariations are proposed in Barndorff-Nielsen and Shephard (2004a), thresholds covariances in Mancini and Gobbi (2012), outlyingness weighted covariances in Boudt et al. (2011b), and disentangled covariances in Boudt et al. (2012).

Second, intraday prices are unreliably recorded, as they do not necessarily correspond to those at which the underlying asset has been traded, as pointed out in Zhou (1996). This phenomenon, labeled as market microstructure noise (noise henceforth), affects significantly the properties of realized measures. Several solutions are provided in the literature both for realized variances and covariances. They include, among others, sparse sampling (Andersen et al. (2001) and Bandi and Russell (2008)), multi-scale estimators (Zhang et al. (2005) and Zhang (2006)), pre-averaging techniques (Podolskij and Vetter (2009), Jacod et al. (2009), Christensen et al. (2010a) and Christensen et al. (2013)), realized kernels (Barndorff-Nielsen et al. (2008) and Barndorff-Nielsen et al. (2011)), pseudo-maximum likelihood techniques (Aït-Sahalia et al. (2010)), and measures based on the Kalman filter and the EM algorithm (Shephard and Xiu (2012) and Corsi et al. (2012)).

Third, and this is specific to the multivariate setup, while price series are non-synchronous and discrete, the underlying theory of realized estimators is based on continuous stochastic processes. As a result, most of the multivariate tools require synchronous data. Several estimators and sampling methods have been proposed to cope with non-synchronous transactions.

This article investigates the properties of the class of disentangled estimators introduced by 
Boudt et al. (2012), i.e. realized covariances computed as the product of realized volatilities and correlations. Though numerous estimators can be constructed on basis of combinations of volatilities and correlations, we focus on estimators presenting robust properties with respect to jumps. In other words, we consider finite activity jumps and build the covariance estimator on basis of robust correlation and volatility estimators.

Our main contribution is to show that this class of estimators, if implemented properly, is appropriate for covariation measurement. Indeed, the estimators are precise, simple to program, computationally fast, and the estimated matrix is positive definite. Moreover, we underline the robustness of the estimator with respect to the three aforementioned issues related to high-frequency data. First, we point out that the use of robust statistics for the correlations, such as Gaussian ranks and Spearman $\rho$, provide jump-robust estimates. Second, we show that microstructure noise does not generate biases when log-returns are pre-averaged. Finally, asynchronous trading does not generate biases when log-returns are aligned using previous-tick interpolations.

The reason for disentangling covariances into correlations and volatilities is the optimal use of the available information. Measuring separately volatilities and correlations allows to measure each component using the largest amount of available information. Indeed, as returns only need to be synchronized for correlations, volatilities are measured using the full sample of data. This approach has potential advantages in terms of precision for the estimation. In fact, separating the estimation of correlations and volatilities is not an uncommon practice in econometrics. In the parametric MGARCH set up, Bollerslev (1990), Tse and Tsui (2000), and Engle (2002) propose equivalent approaches with CCC and DCC models (see Bauwens et al. (2006) for an extensive review). Halbleib and Voev (2011) propose a mixed approach, combining the DCC for the correlations and the realized estimators for the volatilities.

We compare different combinations of estimators and study their properties in a Monte Carlo exercise and with real data.

The Monte Carlo study is based on four different models that are frequently used in the literature. For testing our estimators in a realistic setting, these models are simulated along with different components to accommodate finite activity jumps, microstructure noise, and asynchronous trading. We find out that the pre-averaged version of disentangled realized covariances computed with Gaussian ranks and median-based realized volatilities provide the most precise results in case of jumping assets and it competes closely with realized kernels in absence of jumps.

We empirically assess the goodness of disentangled realized covariances through an indirect evaluation based on a minimum variance portfolio management exercise. Data represent the largest companies traded on the NYSE. Competing estimators are also evaluated in the application. We find that disentangled realized covariances can be used reliably with forecasting models such as the HEAVY of Noureldin et al. (2012). Finally we underline different empirical implications from 
the use of different forecasting models.

Throughout the paper, we use the following notations, unless explicitly stated otherwise: i) $p$ denotes the dimension of the random vector of returns, which has a covariance matrix with elements generically indexed by $i$ and $j$, ii) $t$ denotes time (measured in low frequency, typically a day), iii) $N$ is the number of high frequency observations (intraday observations if $t$ is measured in days) with index $m$ (i.e. $m=0, \ldots, N$ ), iv) every day is divided in $K$ blocks or subsamples indexed by $q$ (i.e. $q=1, \ldots, K)$, and $k$ is the number of observations in each block. The hierarchy of frequency is therefore: 1 day composed by $N$ intraday observations, divided in $K$ blocks with $k$ observations within each block. Bold denotes vectors and matrices.

The rest of the paper is organized as follows. In Section 2 we first introduce the data generating process, notation, and the class of disentangled estimators. In Section 3, we define several synchronization schemes. We report the results of a Monte Carlo study based on various models in section 4 . Section 5 presents the gains of the disentangled estimators in terms of returns on investment. Section 6 concludes. Additional results are reported in the Appendix.

\section{Jump-robust covariation measurement}

We consider a $p$-dimensional random vector of no-arbitrage log-prices denoted $\left\{\mathbf{X}_{t}\right\}_{t \geq 0}$ and defined on a filtered probability space $\left(\Omega, \mathcal{A},\left(\mathcal{A}_{t}\right)_{t \in[0,1]}, \mathcal{P}\right)$. We assume that the process is adapted to the filtration $\left(\mathcal{A}_{t}\right)_{t \in[0,1]}$ and that the vector of log-prices behaves as an Itō semimartingale with finite activity jumps:

$$
\mathbf{X}_{t}=\int_{0}^{t} \boldsymbol{\mu}_{u} d \mathbf{u}+\int_{0}^{t} \boldsymbol{\Lambda}_{u} d \mathbf{W}_{u}+\sum_{0 \leq s \leq t} \mathbf{J}_{s}
$$

The process $\boldsymbol{\mu}_{u}$ is locally element-wise bounded predictable, and the elements of $\boldsymbol{\Lambda}_{u}$ are adapted cádlag processes such that $\boldsymbol{\Sigma}_{u}=\boldsymbol{\Lambda}_{u}^{T} \boldsymbol{\Lambda}_{u}$. The matrix $\boldsymbol{\Sigma}_{u}$ denotes the spot (or instantaneous) covariance matrix of the process. The random vector $\mathbf{W}_{u}$ denotes a $p$-dimensional standard Brownian motion and $\mathbf{J}_{s}$ denotes the jumps magnitude. Jumps are driven by a finite activity counting process $\boldsymbol{N}_{\boldsymbol{t}}$ such that $E\left[\boldsymbol{N}_{\boldsymbol{t}}\right]<\infty$. The component $\int_{0}^{t} \boldsymbol{\mu}_{u} d \mathbf{u}+\int_{0}^{t} \boldsymbol{\Lambda}_{u} d \mathbf{W}_{u}$ in (1) is the continuous part and denoted by $\mathbf{X}_{t}^{c}$, so that

$$
\mathbf{X}_{t}=\mathbf{X}_{t}^{c}+\sum_{0 \leq s \leq t} \mathbf{J}_{s}
$$

Since we are interested in robustness to large unexpected deviations in asset prices, we limit to finite activity jumps driven by a Poisson process as described in (1), despite the fact that infinite activity jumps processes are also considered in practice (e.g. Carr et al. (2002)). Further 
work might integrate finite and infinite activity jumps, and test whether it is possible to separate between large and small jumps in the price process using the statistics presented hereafter.

The period of interest is $[0,1]$ (e.g. one day). We denote by $\pi_{N}$ an ordered set of times such that $0=t_{0}<t_{1}<\ldots<t_{m}<\ldots<t_{N}=1$ forming a partition (see definition 1 below) of the period considered, and $\mu\left(\pi_{N}\right)$ is the mesh of the partition. In this setup, the $\pi_{N}$-quadratic variation process of $\left\{\mathbf{X}_{t}\right\}_{t \geq 0}$ is defined as the random process

$$
\mathbf{Q}_{\pi_{N}}\left(\mathbf{X}_{t}\right)=\sum_{m=1}^{N}\left(\mathbf{X}_{t_{m} \wedge t}-\mathbf{X}_{t_{m-1} \wedge t}\right)\left(\mathbf{X}_{t_{m} \wedge t}-\mathbf{X}_{t_{m-1} \wedge t}\right)^{T}
$$

If $\mathbf{Q}_{\pi_{N}}\left(\mathbf{X}_{t}\right)$ converges in probability to a process $\left\{\mathbf{V}_{t}\right\}_{t \in[0,1]}$ for any sequence partition $\pi_{N}$ over the interval $[0,1]$ such that $\boldsymbol{\mu}\left(\pi_{N}\right) \rightarrow 0$ as $N \rightarrow \infty$, then we call $\left\{\mathbf{V}_{t}\right\}_{t \in[0,1]}$ the quadratic variation, and denote it by $[\mathbf{X}]_{t}$. Assuming $\mathbf{X}_{0}=0$, it is well known for Itō semimartingales that

$$
[\mathbf{X}]_{t}=[\mathbf{X}]_{t}^{c}+\sum_{0 \leq s \leq t} \Delta \mathbf{J}_{s} \Delta \mathbf{J}_{s}^{T}
$$

where $[\mathbf{X}]_{t}^{c}=\int_{0}^{t} \boldsymbol{\Sigma}_{u} d \mathbf{u}$ is the "path-by-path" continuous part of the quadratic variation (Protter (2004)), which also corresponds to the quadratic variation of the continuous part $\mathbf{X}_{t}^{c}$. The $\pi_{N^{-}}$ quadratic variation process $\mathbf{Q}_{\pi_{N}}\left(\mathbf{X}_{t}\right)$ can therefore be seen as a finite sample measure of risk composed of two sources: risks related to the Brownian component and those related to the finite activity jumps part.

Considering $\sigma_{u}^{i, j}$ as the $i$-th row and $j$-th column element of $\boldsymbol{\Sigma}_{u}$, we have that $\sigma_{u}^{i, j}=\rho_{u}^{i, j} \sigma_{u}^{i} \sigma_{u}^{j}$, where $\sigma_{u}^{i}$ and $\sigma_{u}^{j}$ are the spot volatilities and $\rho_{u}^{i, j}$ is the spot correlation. Our object of interest is the estimation of $[\mathbf{X}]_{1}^{c}$ by estimating separately the elements $\rho_{u}^{i, j}, \sigma_{u}^{i}$ and $\sigma_{u}^{j}$

$$
[X]_{1}^{c, i, j}=\int_{0}^{1} \rho_{u}^{i, j} \sigma_{u}^{i} \sigma_{u}^{j} d u .
$$

In other words, we are interested in a jump robust estimator of the daily integrated covariation by considering separately spot correlations and volatilities. As mentioned above, this approach allows to use the full data sample for volatilities as no synchronization technique is required for their estimation.

We now define more rigorously the partitions one might encounter: ${ }^{1}$

Definition 1 Let $\pi_{N_{i}}:=\left[0=t_{0}^{i}<t_{1}^{i}<\ldots<t_{m}^{i}<\ldots<t_{N_{i}-1}^{i}<t_{N_{i}}^{i}=1\right]$ be the partition on the time interval $[0,1]$ for asset $i$. Likewise for asset $j$. We say that $\pi_{N_{i}}$ and $\pi_{N_{j}}$ are

1. synchronous and evenly spaced if $N_{i}=N_{j}, \pi_{N_{i}}=\pi_{N_{j}}$ and $t_{m}^{i}-t_{m-1}^{i}=t_{m}^{j}-t_{m-1}^{j}=\frac{1}{N}$.

2. synchronous and evenly spaced if $N_{i}=N_{j}, \pi_{N_{i}}=\pi_{N_{j}}$ but time intervals between prices are not deterministic.

\footnotetext{
${ }^{1}$ This definition is in terms of 2 assets, but it can be generalized to any dimension.
} 
3. asynchronous if $\pi_{N_{i}} \neq \pi_{N_{j}}$ but we might have that $\pi_{N_{i}} \cap \pi_{N_{j}} \neq\{0,1\}$, i.e. there may be common points in the two sets except the first and the last (both sets are partitions of the same interval).

4. completely asynchronous if $\pi_{N_{i}} \neq \pi_{N_{j}}$ and $\pi_{N_{i}} \cap \pi_{N_{j}}=\{0,1\}$.

Figure 1 provides a diagrammatic representation of the partitions. The upper two lines corresponds to a partition of type 1 . The arrival times are regularly spaced and synchronized. The next two lines represent the partition of type 2 , in which arrival times are also synchronized but irregularly spaced. The bottom half of the figure represents the partitions 3 and 4 in which obser-

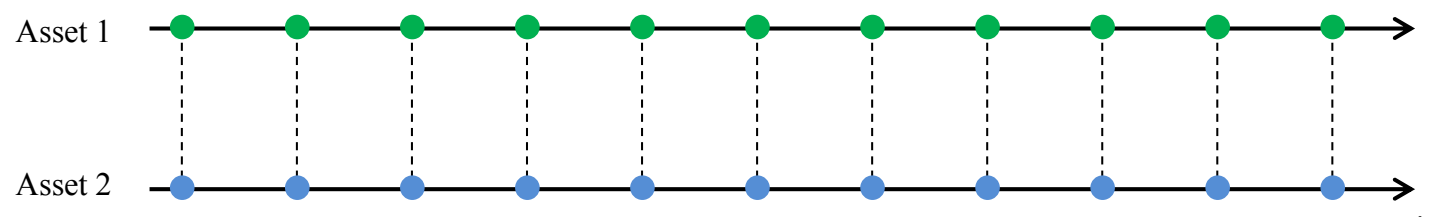

Time

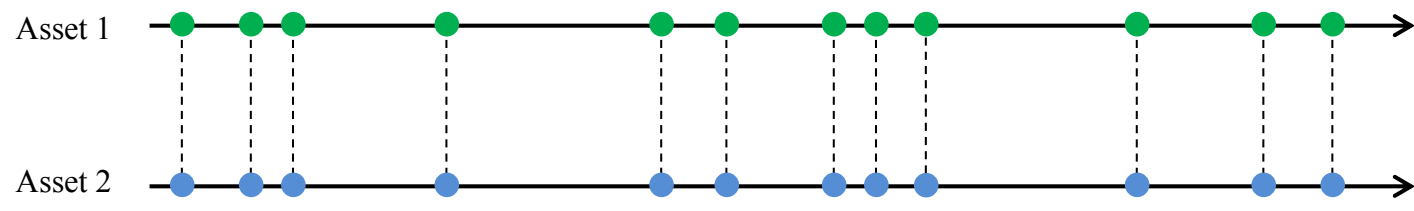

Time

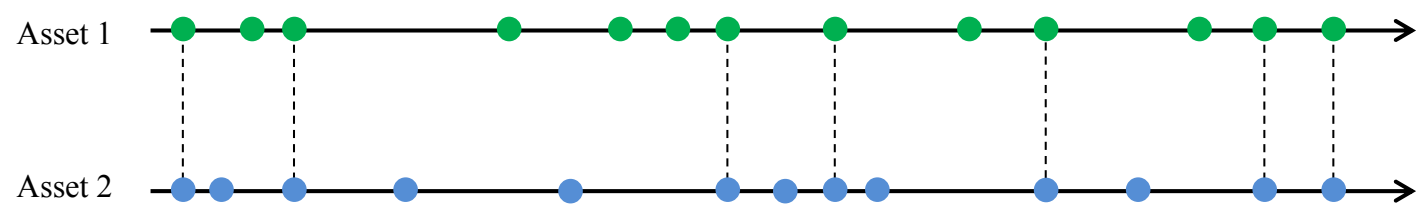

Time

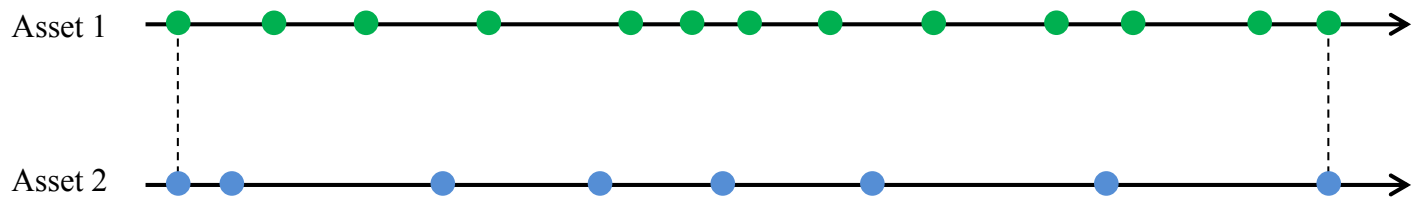

Time

The upper two lines corresponds to a partition of type 1. The arrival times are regularly spaced and synchronized. The next two lines represent the partition of type 2, in which arrival times are also synchronized but irregularly spaced. The bottom half of the figure represents the partitions 3 and 4 in which observations are asynchronized, though in partition 3 there maybe sporadic common arrival times, an event excluded in partition 4 .

In order to introduce the class of estimators in a clear way, we first assume that $\pi_{N_{i}}$ and $\pi_{N_{j}}$ are of type 1 . The time intervals are denoted as $\Delta_{m}^{N} t=t_{m}-t_{m-1}$ and equal to $1 / N$ under type 1. Let $\Delta_{m}^{N} \mathbf{X}=\mathbf{X}_{m / N}-\mathbf{X}_{(m-1) / N}=\left(X_{i, m / N}-X_{i,(m-1) / N}, X_{j, m / N}-X_{j,(m-1) / N}\right)$ be the vector 
of synchronous log-returns computed over the period of interest. ${ }^{2}$ The returns scaled (by time) are $\Delta_{m}^{N} \mathbf{X}^{*}=\left(\Delta_{m}^{N} t\right)^{-\frac{1}{2}} \Delta_{m}^{N} \mathbf{X}=\sqrt{N} \Delta_{m}^{N} \mathbf{X}^{3}$

We construct $K$ non-overlapping smaller subsets (or blocks) of returns containing each $k$ data points, i.e. $N=K \times k{ }^{4}$ We denote by $B_{q}^{k} \mathbf{X}^{*}=\left(\Delta_{m}^{N} \mathbf{X}^{*}\right)_{(q-1) k+1 \leq m \leq q k}$ the subset of scaled returns contained in block $q$.

The class of Disentangled Realized Covariances (DRC) between assets $i$ and $j$ is

$$
D R C^{i, j}(K)=\frac{1}{K} \sum_{q=1}^{K} r^{i, j}\left(B_{q}^{k} \mathbf{X}^{*}\right) v^{i}\left(B_{q}^{k} \mathbf{X}^{*}\right) v^{j}\left(B_{q}^{k} \mathbf{X}^{*}\right),
$$

where $r^{i, j}\left(B_{q}^{k} \mathbf{X}^{*}\right), v^{i}\left(B_{q}^{k} \mathbf{X}^{*}\right)$ and $v^{j}\left(B_{q}^{k} \mathbf{X}^{*}\right)$ denote respectively estimators of the correlation and volatilities for assets $i$ and $j$ computed using the scaled returns contained in block $q$. The choice of these estimators determine the properties of (5).

Indeed, many choices are available for $r^{i, j}\left(B_{q}^{k} \mathbf{X}^{*}\right), v^{i}\left(B_{q}^{k} \mathbf{X}^{*}\right)$ and $v^{j}\left(B_{q}^{k} \mathbf{X}^{*}\right)$. In this article we select some and test them. The methodology we use is somewhat inductive. We begin by analyzing the goodness of combinations of estimators for volatilities and correlations with Monte Carlo simulations. Then, on basis of the finite sample results, we select the best estimator and compare its performances with benchmark jump-robust estimators from the literature. Our approach therefore extends Boudt et al. (2012) as several combinations are considered, different sampling schemes are used, and noise is introduced.

We close this sub-section with three remarks. The first concerns consistency and the jumprobust properties of the estimators. They are related to the idea that, in a small interval, logreturns generated by the process in (1) can be well approximated by a Brownian motion with constant covariance matrix: if $(q-1) k+1 \leq m \leq q k$ then $\Delta_{m}^{N} \mathbf{X} \approx \Lambda_{\frac{(q-1) k+1}{N}} \Delta_{m}^{N} \mathbf{W}$ where the subindex $\frac{(q-1) k+1}{N}$ corresponds to the starting time of block $q$ (see e.g. Mykland and Zhang (2009)). Since the approximated contiguous scaled returns located in block $q$ display constant spot covariance matrix and have distribution $N\left(0, \boldsymbol{\Sigma}_{\frac{(q-1) k+1}{N}}\right)$, consistent robust estimators under Gaussianity enable to estimate the spot covariance matrix in each block. The average over blocks provides an estimator of the integrated covariance matrix that intuitively maps to a Riemann sum over time intervals.

Second, the finite activity jumps that we consider correspond to large unexpected movements. In a small block, these jumps can be mapped intuitively to outliers in the statistical sense. Estimators that are robust to outliers thus eliminate the effect of finite activity jumps.

Third, in the case of synchronous data $\left(\pi_{N_{i}}\right.$ and $\pi_{N_{j}}$ are of types 1 or 2 in definition 1$)$, measuring volatilities and correlations separately has of course little interest since no efficiency

\footnotetext{
${ }^{2}$ Note that since partitions are of type $1, \mathbf{X}_{t_{m}}=\mathbf{X}_{m / N}$.

${ }^{3}$ For other types of partitions, $\Delta_{m}^{N} t$ is a random quantity and can induce spurious random fluctuation effects.

${ }^{4}$ In some instances the last block may have a different amount of points depending on the initial amount of observations and the amount of blocks.
} 
can be gained from disentangled estimation. Our class of estimator is best suited in the case of partitions $\pi_{N_{i}}$ and $\pi_{N_{j}}$ that are of type 3 or 4 , requiring a synchronization technique to estimate the spot correlations within each block. We discuss this point more in detail later.

\subsection{Estimation of the spot volatilities}

In this section, we describe two jump-robust estimators of spot volatilities. They are derived from two classes of integrated volatility estimators proposed by Christensen et al. (2010b) and Andersen et al. (2012). We limit the analysis to these two classes as both are based on a blocking strategy, and hence map easily into the class of disentangled realized covariances.

The estimator of integrated volatility proposed by Christensen et al. (2010b) is based on quantiles and defined, for asset $i$, as

$$
\operatorname{QRV}^{i}(\lambda, K)=\frac{1}{K} \sum_{q=1}^{K} \frac{s_{i}\left(B_{q}^{k} \mathbf{X}^{*}, \lambda\right)}{\nu(\lambda)},
$$

where $s_{i}\left(B_{q}^{k} \mathbf{X}^{*}, \lambda\right)=g_{\lambda k}^{2}\left(B_{q}^{k} \mathbf{X}^{*}\right)+g_{(1-\lambda) k+1}^{2}\left(B_{q}^{k} \mathbf{X}^{*}\right)$ and the function $g_{k}(\mathbf{x})=\mathbf{x}_{(k)}$ denotes the $k$-th order statistics. The parameter $\lambda$ is the probability level at which QRV is computed. ${ }^{5}$ The term $\nu(\lambda)$ in $(6)$ is a scaling factor given by $\nu(\lambda)=\mathbb{E}\left[\left|U_{(\lambda m)}\right|^{2}+\left|U_{(m-\lambda m+1)}\right|^{2}\right]$, where $U_{(\lambda m)}$ is the $\lambda m$-th order statistics of a sample of $m$ i.i.d. normal random variables $\left(U_{1}, \ldots, U_{m}\right)$. This scaling can be computed by simulation and ensures consistency of the estimator under Gaussianity.

The second class of estimators for integrated volatility is a generalization of those proposed by Andersen et al. (2012), which, for asset $i$, are defined as

$$
\begin{aligned}
\operatorname{MinRV}_{K}^{i} & =\frac{1}{\xi^{\operatorname{Min}}(k)} \sum_{q=1}^{K} \min \left(\left|B_{q}^{k} \mathbf{X}^{*}\right|\right)^{2} \text { and } \\
\operatorname{MedRV}_{K}^{i} & =\frac{1}{\xi^{\operatorname{Med}}(k)} \sum_{q=1}^{K} \operatorname{med}\left(\left|B_{q}^{k} \mathbf{X}^{*}\right|\right)^{2} .
\end{aligned}
$$

The scalings $\xi^{\text {Min }}(k)$ and $\xi^{\text {Med }}(k)$ are such that the summands are consistent estimators of the spot volatility in the corresponding block under the assumption that observations are i.i.d. Gaussian. These constants are functions of the number of observations per block. Andersen et al. (2012) consider $k$ equal to 2 and 3 for $\operatorname{MinRV}_{N}^{i}$ and $\operatorname{MedRV}_{N}^{i}$ respectively, for which the scalings have closed-form expressions. For other values of $k$, they may have to be computed by simulations. ${ }^{6}$ If $k=1$, the scaling is trivially equal to 1 for both estimators, which coincide with the baseline realized variance estimator. If $k \rightarrow \infty$, the scaling for MedRV converges to

\footnotetext{
${ }^{5}$ The information contained at more than one probability level can be exploited by considering $Q R V_{N}^{i}(\underline{\lambda})=$ $\alpha^{T} \underline{Q R V_{N}^{i}}(\underline{\lambda})$, where $\alpha$ is a vector (summing to one) that has the same size as $\underline{\lambda}$.

${ }^{6}$ Boudt et al. (2012) use $k=5$ for the MedRV and a scaling of 1.624 .
} 
2.198, while the scaling for MinRV increases exponentially to infinity. ${ }^{7}$ Because of this drawback, combined with its sensitivity to zero returns (leading to a bias towards zero), we do not consider the MinRV estimator. MedRV by contrast strikes a good balance between stability and jump robustness.

Based on $\operatorname{QRV}^{i}(\lambda, K)$ and $\operatorname{MedRV}_{K}^{i}$, the estimators for the spot volatility $v^{i}\left(B_{q}^{k} \mathbf{X}^{*}\right)$ we consider are

$$
\begin{aligned}
\operatorname{QRV}_{k, q}^{i}(\lambda) & =\frac{s\left(B_{q}^{k} \mathbf{X}^{*}, \lambda\right)}{\nu(\lambda)} \text { and } \\
\operatorname{MedRV}_{k, q}^{i} & =\frac{\operatorname{med}\left(\left|B_{q}^{k} \mathbf{X}^{*}\right|\right)^{2}}{\xi^{M e d}(k)}
\end{aligned}
$$

And likewise for $v^{j}\left(B_{q}^{k} \mathbf{X}^{*}\right)$.

\subsection{Estimation of the spot correlations}

The statistical literature on robust estimators for correlations is extensive (see e.g. Shevlyakov and Smirnov (2011) and references therein). We measure spot correlations using benchmarks of this literature (Kendall's $\tau$ and Spearman's $\rho$ ), as well as other alternatives - quadrant signs and Gaussian ranks - that deliver good results in the realized literature (Boudt et al. (2012)).

To facilitate notations in this section, we denote $L_{q}=(q-1) k+1$ and $U_{q}=q k$ the lower and upper bounds for the index of returns belonging to $B_{q}^{k} \mathbf{X}^{*}$.

Kendall's $\tau$ is based on the statistical and geometric properties of elliptical distributions. Heuristically, it considers concordance of the combinations of observations by means of the signs. Kendall's $\tau$ between two random variables $X_{i}$ and $X_{j}$ is defined as

$$
\rho_{\tau}^{i, j}=E\left(\operatorname{sign}\left(X_{i}-\tilde{X}_{i}\right)\left(X_{j}-\tilde{X}_{j}\right)\right)
$$

where $\left(\tilde{X}_{i}, \tilde{X}_{j}\right)$ is an independent copy of $\left(X_{i}, X_{j}\right)$. In our setup, the estimator for block $q$ is

$$
r_{\tau, q}^{i, j}=\frac{2}{k(k-1)} \sum_{L_{q} \leq n<s \leq U_{q}} \operatorname{sign}\left(\left(\Delta_{n}^{N} X_{i}^{*}-\Delta_{s}^{N} X_{i}^{*}\right)\left(\Delta_{n}^{N} X_{j}^{*}-\Delta_{s}^{N} X_{j}^{*}\right)\right) .
$$

The estimated Pearson correlation is obtained as $r_{q}^{i, j}=\sin \left(\frac{\pi}{2} r_{\tau, q}^{i, j}\right)$. It is pairwise and does not necessarily provide positive definite estimated matrices. However, if the sample size is at least three times larger than the cross section, the resulting matrix is positive definite with probability one (Boudt et al. (2011a)).

Spearman's rho is based on the Pearson correlation between cumulative distribution functions. If one defines $F_{i}(x)=P\left(X_{i} \leq x\right)$ (and likewise for $X_{j}$ ), the Spearman's correlation is

$$
\rho_{S p}^{i, j}=\rho^{i, j}\left(F_{i}\left(X_{i}\right), F_{j}\left(X_{j}\right)\right) .
$$

\footnotetext{
${ }^{7}$ The constant 2.198 is the square of 1.483 , which is the scaling factor of the median absolute deviation in an i.i.d. Gaussian setup (Rousseeuw and Croux (1993)).
} 
The estimator for block $q$, denoted $r_{S p, q}^{i, j}$, is the sample Pearson correlation between ranks of the vectors in $B_{q}^{k} \mathbf{X}^{*}$. The estimated Pearson correlation between $X_{i}$ and $X_{j}$ is then $r_{q}^{i, j}=$

$2 \sin \left(\frac{\pi}{6} r_{S p, q}^{i, j}\right)$. The estimated matrix is positive definite with probability one if the sample size is at least two times larger than the cross sectional size (Boudt et al. (2011a)).

Quadrant signs, or quadrant correlations are defined as

$$
\rho_{Q d}^{i, j}=E\left(\operatorname{sign}\left(X_{1}-\operatorname{median}\left(X_{1}\right)\right)\left(X_{2}-\operatorname{median}\left(X_{2}\right)\right)\right) .
$$

The estimator based on $B_{q}^{k} \mathbf{X}^{*}$ is the sample average of the signs

$$
r_{Q d, q}^{i, j}=\frac{1}{k} \sum_{n=L_{q}}^{U_{q}} \operatorname{sign}\left(\left(\Delta_{n}^{N} X_{i}^{*}-\operatorname{median}\left(\Delta_{n}^{N} X_{i}^{*}\right)\right)\left(\Delta_{n}^{N} X_{j}^{*}-\operatorname{median}\left(\Delta_{n}^{N} X_{j}^{*}\right)\right)\right) .
$$

The estimated Pearson correlations and the condition for positive definite estimated matrix are the same as for the Kendall's $\tau$.

Gaussian ranks is a direct estimator of the Pearson correlation:

$$
r_{\Phi, q}^{i, j}=\frac{1}{\psi_{k}} \sum_{n=L_{q}}^{U_{q}} \Phi^{-1}\left(\frac{\operatorname{rank}\left(\Delta_{n}^{N} X_{i}^{*}\right)}{k+1}\right) \Phi^{-1}\left(\frac{\operatorname{rank}\left(\Delta_{n}^{N} X_{j}^{*}\right)}{k+1}\right),
$$

where $\psi_{k}=\sum_{n=1}^{k} \Phi^{-1}\left(\frac{n}{k+1}\right)^{2}$ only depends on the amount of points $k$ in block $q, \Phi^{-1}(\cdot)$ denotes the quantile function of the standard normal distribution, and $\operatorname{rank}\left(\Delta_{n}^{N} X_{i}^{*}\right)$ the rank of $\Delta_{n}^{N} X_{i}^{*}$ in block $q$. Positive semi-definiteness is ensured as long as the sample size is greater than the cross section.

\subsection{Positive definiteness and number of blocks}

The matrix version of (5) is

$$
\mathbf{D R C}(K)=\frac{1}{K} \sum_{q=1}^{K} \mathbf{S}\left(B_{q}^{k} X^{*}\right) \mathbf{R}\left(B_{q}^{k} X^{*}\right) \mathbf{S}\left(B_{q}^{k} X^{*}\right),
$$

where $\mathbf{R}(\cdot)$ denotes the jump robust estimator of the spot correlation matrix and $\mathbf{S}(\cdot)$ is a diagonal matrix containing jump robust estimates of the spot volatilities of each asset over block $q$. Positive definiteness of $\mathbf{D R C}(K)$ depends on $\mathbf{R}(\cdot)$ and $\mathbf{S}(\cdot)$. If $\mathbf{R}(\cdot)$ is positive definite, and if the diagonal matrix $\mathbf{S}(\cdot)$ has no zero entries, then $\mathbf{S}(\cdot) \mathbf{R}(\cdot) \mathbf{S}(\cdot)$ and the average (9) are positive definite.

In practice, choices have to be made for the number of blocks $K$ (and hence the number of observations per block $k$ ), which, for a fixed sample size $N$, lead to the classical trade-off between precision and bias. A smaller amount of blocks implies a larger amount of available observations for the estimation. In the opposite, estimation based on a larger amount of blocks allows to 
decrease the sensitivity to zero-returns, reducing the chances of downward biases. Moreover, the spot correlations and volatilities being time-varying, inference based on a small amount of blocks is unlikely to capture accurately the dynamics, advocating the use of a larger number of blocks.

The amount of blocks per day should be an decreasing function of the cross section size $p$. In higher dimensions, smaller amount of blocks should be used in order to preserve positive definiteness. Note that this points in favor of the estimation of $\mathbf{R}(\cdot)$ on basis of Gaussian ranks, which provide positive definite matrices as long as the amount of assets considered is smaller than the sample size. On basis of simulated and real data, a reasonable user choice for frequently sampled data is around 5 blocks.

\section{Market Microstructure noise}

Up to this point, we described the class of DRC estimators under the assumption that efficient prices are observable and not contaminated by market microstructure noise. In practice, however, observed prices do not always match with those that are exchanged on markets (see e.g. Zhou (1996)). From a modeling viewpoint, noise is an additive component to log-prices that can be written as $Y_{t}=X_{t}+\eta_{t}$, where $X_{t}$ denotes the efficient log-prices (1) and $\eta_{t}$ denotes the market microstructure noise. Generally, noise generates upward biases in standard realized volatilities and is less impactful on covariance measures. We describe three techniques for decreasing the impact of noise.

Sparse sampling consists of sampling prices on a sparse grid of time points, i.e. choosing a value for $\Delta$ in the interpolation scheme. At lower frequencies, the impact of noise is known to be less relevant and the bias tends to vanish (Barndorff-Nielsen and Shephard (2007)). The resulting estimator is computed using the synchronous low frequency data. Sparse sampling may reduce significantly the number of available observations, which has two drawbacks: it limits the size of the cross section for which the estimated matrix is positive definite, and the precision of the estimates worsens.

Subsampling is introduced in the univariate case by Zhang et al. (2005) and Zhang (2006), and studied in the multivariate setup by Zhang (2011) and Boudt and Zhang (2013) among others. Instead of using contiguous non-overlapping blocks of returns, subsampling uses overlapping subsamples containing returns sampled at a lower frequency. The successive estimates are then averaged.

McAleer and Medeiros (2008) provide the following intuitive example. If one has noisy 1second returns and wishes to use subsampling with 5 -minute returns, she can compute returns 
using log-prices recorded on the following grid of time coordinates [9:30-9:35], [9:35-9:40], [9:409:45], ..., [15:55-16:00]. The remaining unused points are used to construct new series of returns. For example, starting ten seconds later we have the new grid [9:30:10-9:35:10], [9:35:10-9:40:10], $[9: 40: 10-9: 45: 10], \ldots,[15$ 50:10-15:55:10]. The new series are used to replace blocks and estimate the covariance.

We construct $K$ overlapping sub-grids at a calendar frequency $\delta$, i.e. we skip $\delta$ points in $\pi_{N_{i}}$ between two consecutive points of the new sub-grid. Returns are computed from prices projected on the sparse grid of times using previous-tick interpolation. They are denoted by $\Delta_{m, q}^{N} \mathbf{Y}^{*}=\sqrt{N / \delta}\left(\mathbf{Y}_{\frac{(q-1)+\delta m}{N}}-\mathbf{Y}_{\frac{(q-1)+\delta(m-1)}{N}}\right)$ where $q=1, \ldots, K, m=1, \ldots,\lfloor(N-q+1) / \delta\rfloor$, and Ł.」 denotes the floor operator. ${ }^{8}$ We denote the new set of returns contained in subsample $q$ by $B_{q}^{\delta} \mathbf{Y}^{*}=\left(\Delta_{m, q}^{N} \mathbf{Y}^{*}\right)_{1 \leq m \leq\lfloor(N-q+1) / \delta\rfloor}$. The estimator is then computed as in (5) by replacing the blocks by the new subsamples.

Sampling at lower frequencies allows to decrease the impact of noise on estimates and averaging over the subsamples allows to increase the efficiency of the estimator. However, the cost of subsampling is that the size of the cross section for which the estimator is positive definite will be limited by the frequency of the subsamples. The reason is alike to sparse sampling since the average is composed of covariance matrices based on low frequency data.

Pre-averaging is introduced by Podolskij and Vetter (2009) and studied by Jacod et al. (2009). Multivariate extensions can be found in Christensen et al. (2010a) and Christensen et al. (2013). It relies on the intuitive idea that if the noise $\eta_{t}$ is i.i.d. with mean zero, then smoothing the $\log$-prices $\mathbf{Y}_{t}$ may decrease the impact of microstructure noise and provide an approximation of the true latent price $\mathbf{X}_{t}$.

We use pre-averaging in calendar time assuming prices are aligned on an homogeneous grid of time. If $\Delta_{m}^{N} \mathbf{Y}^{*}$ denotes the $m$-th vector of noisy scaled returns, pre-averaged returns are defined as

$$
\Delta_{m} \tilde{\mathbf{Y}}^{*}=\frac{1}{\sqrt{k_{N} \psi}} \sum_{j=1}^{k_{N}-1} g\left(\frac{j}{k_{N}}\right) \Delta_{m+j}^{N} \mathbf{Y}^{*}, \text { for } m=0, \ldots, N-k_{N}+1,
$$

where $g($.$) is a kernel function, \psi=\frac{1}{k_{N}} \sum_{j=1}^{k_{N}-1} g^{2}\left(\frac{j}{k_{N}}\right)$ and $k_{N} / \sqrt{N}=\theta+o\left(N^{-1 / 4}\right)$ are computed following Christensen et al. (2010a). The scaling in front of the sum is necessary to avoid insample biases in the estimates. We replace the returns used to construct the blocks in the previous section by their pre-averaged counterpart and compute the estimator following equation (5), which provides the pre-averaged version of the class of DRC. Pre-averaging of log-returns enables to keep more points for the estimation. Consequently, precise estimates can be obtained and the dimension

\footnotetext{
${ }^{8}$ Note that if returns are sampled every second along a grid $\pi_{N_{i}}$ of type 1 , then $K=\delta$.
} 
is not reduced as in the case of sparse sampling.

\section{Synchronization schemes}

We now assume that the partitions $\pi_{N_{1}}$ and $\pi_{N_{2}}$ (see definition 1) are of type 3 or 4 , i.e. observations are asynchronous and assets may have different sample sizes. As in the synchronous case every day there is an opening and a closing, and the day is evenly divided in $K$ blocks. The main difference with regularly spaced arrivals is that that the number of observations per block is random. Once re-scaled, these observations can be used to estimate the volatilities, one by one independently of each other. However, for the estimation of the correlations within each block, observations for all assets need to be synchronized. What follows is the list of the synchronization techniques that we use.

Interpolation is based on first choosing a fixed calendar sampling frequency. For example, it is common in practice to use 5, 10 or 15 minutes returns, i.e. returns computed on basis of prices sampled every 5, 10 and 15 minutes along the day. This is the multivariate extension of sparse sampling (see Andersen et al. (2001)) and it provides homogenous time series (Dacorogna et al. (2001)). The sampling frequency is chosen according to an optimality criterion, such as the minimization of issues related to market microstructure noise and jumps. In any case, the choice of the calendar sampling frequency is delicate and may significantly modify the statistical properties of the estimators. On the one hand, sampling at higher frequencies entails a larger sample and potentially more precise estimates. On the other hand, the impact of microstructure noise is known to be more important at high frequencies and can generate biases in realized measures.

Asynchronous trading induces microstructure effects that can lead to downward bias in the correlations among assets, such as the Epps effect (after Epps (1979)) that is illustrated in Figure 2. It shows the baseline realized correlations (of Andersen et al. (2003)) between Apple and the SPDR S\&P500 ETF on April 302012 and as a function of the sampling frequency. The correlation increases with the sampling interval and reach a stable level for low sampling frequencies, illustrating the Epps effect.

Once the frequency is chosen, homogeneous price vectors are constructed. If we denote by $\Delta t$ the frequency at which we sample returns, the number of observations is $\lfloor$ day length (seconds)/ $\Delta t$ (seconds) . Then, if one denotes by $t_{0}$ the starting time of the day, the $i$-th observation is constructed as $\mathbf{X}_{t_{i}}=\mathbf{X}_{t_{k}}$ where $k=\max \left(\tilde{k} \mid t_{\tilde{k}} \leq t_{0}+i \Delta t\right)$ and $t_{k} \leq t_{0}+i \Delta t \leq t_{k+1}$. I.e. prices are constructed by projecting the closest past observation to the $i$-th point of the time grid. This interpolation is called previous tick.

Alternatively, one may opt for linear interpolation. The construction is similar to the previous 


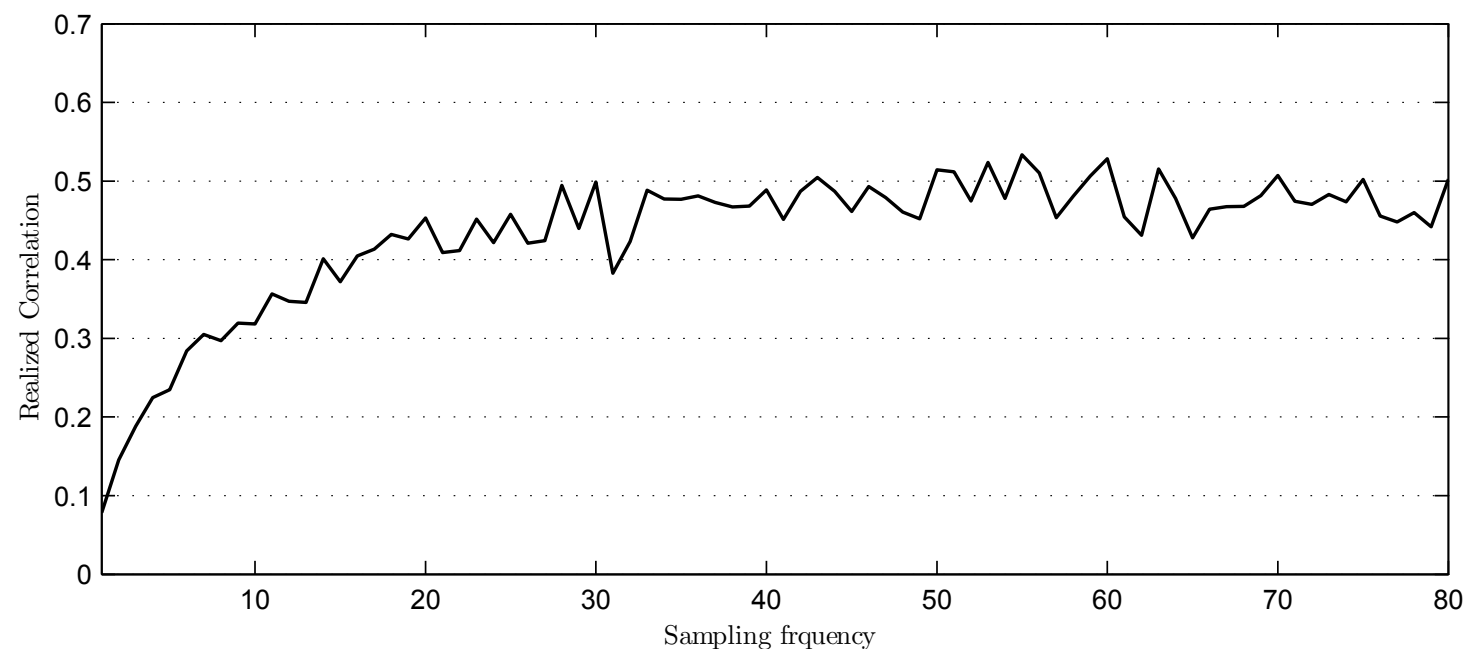

Figure 2: This figure shows an example of microstructure effects induced by asynchronous trading on correlations between SPY and APPL. The x-axis represents the calendar sampling frequency in seconds.

technique and the amount of points in the homogeneous vector is deterministic. If one denotes by $t_{0}$ the starting time of the day, the $i$-th observation is constructed as

$$
X_{t_{i}}=X_{t_{k}}+\frac{t_{0}+i \Delta t-t_{k}}{t_{k+1}-t_{k}}\left(X_{t_{k+1}}-X_{t_{k}}\right)
$$

where $k=\max \left(\tilde{k} \mid t_{\tilde{k}} \leq t_{0}+i \Delta t\right)$.

Refresh time. Barndorff-Nielsen et al. (2011) construct vectors of homogeneous high-frequency prices by projecting asynchronous data on a grid of time coordinates similar to the one used by Harris et al. (1995) and Martens (2004). The new grid of time coordinates has a random amount of data depending on the relative trading intensity/liquidity of the assets considered. As a result, the less liquid asset drives the construction of the grid. Refresh time can in fact being seen as a previous-tick interpolation on a grid of time coordinates defined as follows.

Definition 2 Denote the trading times of an asset $i$ as $t_{1}^{i}, t_{2}^{i}, \ldots$ for $i=1, \ldots, p$ and let $N_{i}^{t}$ be the amount of trades recorded up to time $t$ (with $N_{i}^{1}=N_{i}$ ). Then, the first refresh time is defined as $\tau_{1}=\max \left(t_{1}^{1}, \ldots, t_{1}^{p}\right)$ and the subsequent refresh times as $\tau_{j+1}=\max \left(t_{N_{1}^{\tau_{j}}+1}^{1}, \ldots, t_{N_{p}^{\tau_{j}+1}}^{p}\right)$.

The time $\tau_{1}$ designates the first moment at which all the assets are traded at least once, i.e. the first time at which all prices were refreshed. Then, $\tau_{2}=\max \left(t_{N_{1}^{\tau_{1}}+1}^{1}, \ldots, t_{N_{p}^{\tau_{1}}+1}^{p}\right)$ and, from $\tau_{1}$, we have that $N_{i}^{\tau_{1}} \geq 1 \forall i \in\{1,2, \ldots, p\}$. Moreover, if $\max \left(t_{1}^{1}, \ldots, t_{1}^{p}\right)=t_{1}^{i}$ then $i$ is such that $N_{i}^{t_{1}^{i}}=1$. Thus, we have that $N_{i}^{\tau_{1}}+1 \geq 2 \forall i \in\{1,2, \ldots, p\}$ and $\min \left(N_{1}^{\tau_{1}}+1, \ldots, N_{p}^{\tau_{1}}+1\right)=2$. Intuitively $\tau_{2}$ is the first time after $\tau_{1}$ at which all the assets are traded again. This can be recursively applied for $\tau_{3}, \tau_{4}, \ldots$ up to the end of the sample. The sample size after synchronization is random and 
large cross sections induce more complex computations and increase the risk of deleting a lot of observations.

Refinements have been proposed in the literature. Fan et al. (2012) use a pairwise version of refresh time ("pairwise-refresh" instead of "all-refresh"). This approach has the advantages of retaining more observations. However, it does not provide semi-positive definite matrices, as pointed out by the authors. Hautsch et al. (2012) rank stocks according to their relative liquidity and apply refresh-time to estimate high-dimensional realized kernels, coupled with blocking and regularization techniques related to random matrix theory.

Hayashi and Yoshida (2005)'s scheme handles asynchronous data without projecting prices. As a result, all prices are used in the computation of realized covariances. This scheme was first used to compute realized covariances by aggregating returns recorded in overlapping time intervals. The estimator of cumulative covariance between asset $i$ and $j$ is defined as follows:

$$
\mathbf{R C}_{H Y}^{i, j}=\sum_{m=1}^{N_{i}} \sum_{n=1}^{N_{j}} \Delta_{i, m}^{N_{i}} \mathbf{X} \Delta_{j, n}^{N_{j}} \mathbf{X} \mathbf{1}_{\left\{\left(t_{m-1}^{i}, t_{m}^{i}\right] \cap\left(t_{n-1}^{j}, t_{n}^{j}\right] \neq \emptyset\right\}},
$$

where $\mathbf{1}_{\left\{\left(t_{m-1}^{i}, t_{m}^{i}\right] \cap\left(t_{n-1}^{j}, t_{n}^{j}\right] \neq \emptyset\right\}}$ is an indicator function. The aggregation scheme can be used with different versions of the cumulative covariance estimator as, for example, the thresholds realized covariances of Mancini and Gobbi (2012). However, the class of DRC does not map naturally into the construction of aggregated returns and hence we only apply it to the realized covariances of Andersen et al. (2003) and the thresholds realized covariances of Mancini and Gobbi (2012). More details are provided in the next section.

\section{Monte Carlo Simulation}

We assess the finite sample behavior of the combinations of realized volatilities, correlations and synchronization techniques. We report the performances and compare the best combinations with five competing estimators: the baseline realized covariance ( $\mathrm{RC}$ henceforth) of Andersen et al. (2003), realized bi-power covariation (BPC) of Barndorff-Nielsen and Shephard (2004a), realized outlyingness weighted covariance (OWC) of Boudt et al. (2011b), the estimators based on thresholds (TC) of Mancini and Gobbi (2012), and realized kernels (RK) of Barndorff-Nielsen et al. (2011).

We implement these estimators them as suggested by the authors, which represents the current state of the art. For instance, TC is estimated with a hreshold value $r_{h, t}=9 \mathrm{BPV}_{t} \Delta_{t}^{0.98}$, following Jacod and Todorov (2009), where $\mathrm{BPV}_{t}$ is the bipower variation and $\Delta_{t}$ refers to the time interval between two successive returns. We implement $\mathrm{OWC}$ with hard rejection functions and a threshold of 0.999 , following the results of Boudt et al. (2011b). As for RK, we use refresh time and 
subsampled realized variances to compute the optimal bandwidth. When necessary more details are given below.

In a nutshell, the conclusion of our study is that across the four simulated models (presented below) with jumps, noise and asyncrhonous prices, pre-averaged DRC implemented with Gaussian ranks provide the best results.

\subsection{Data generating processes}

We simulate 10000 trading days from four models that are often used in the literature: a Brownian motion, the Heston model, a stochastic volatility with constant correlation, and a continuous GARCH diffusion. Assuming that the market opens 252 days per year and 6.5 hours per day, a trading day has 23400 seconds. This is the number of prices we generate per day (using the Euler discretization scheme) which implies that 1 second corresponds to $1 /(252 \times 23400)$ units of time.

In the sequel of this section we first show the models. The calibration is done following the choices made in previous works (see Table 1). Next, results are divided in four sub-sections. We start with the ideal world where assets trade synchronously and without noise (section 5.2). Then we introduce asynchronicity (5.3), noise (5.4), and asynchronicity and noise (5.5). ${ }^{9}$

Table 1: Calibration choices

\begin{tabular}{|c|c|c|c|c|c|c|c|c|c|}
\hline \multicolumn{10}{|c|}{ Model 1} \\
\hline & $\sigma_{i}$ & $\rho$ & & & & & & & \\
\hline $\mathrm{i}=1$ & 0.15 & 0.3 & & & & & & & \\
\hline$i=2$ & 0.45 & & & & & & & & \\
\hline \multicolumn{10}{|c|}{ Model 2 - Aït-Sahalia et al. (2010) } \\
\hline & $\mu_{i}$ & $\kappa_{i}$ & $\lambda_{i}$ & $\bar{\sigma}_{i}^{2}$ & $s_{i}$ & $\theta_{i}$ & $\eta_{i}$ & $\rho_{i}$ & $\rho$ \\
\hline $\mathrm{i}=1$ & 0.05 & 3 & 12 & 0.16 & 0.8 & -5 & 0.8 & -0.6 & 0.5 \\
\hline$i=2$ & 0.03 & 2 & 36 & 0.09 & 0.5 & -6 & 0.5 & -0.75 & \\
\hline \multicolumn{10}{|c|}{ Model 3 - Barndorff-Nielsen et al. (2011) } \\
\hline & $\mu_{i}$ & $\beta_{0 i}$ & $\beta_{1 i}$ & $\alpha_{i}$ & $\rho_{i}$ & & & & \\
\hline $\mathrm{i}=1$ & 0.03 & $-5 / 6$ & $1 / 8$ & $-1 / 40$ & 0.5 & & & & \\
\hline $\mathrm{i}=2$ & 0.03 & $-5 / 6$ & $1 / 8$ & $-1 / 40$ & 0.5 & & & & \\
\hline \multicolumn{10}{|c|}{ Model 4 - Voev and Lunde (2007) and Andersen and Bollerslev (1998) } \\
\hline & $k_{i}$ & $\theta_{i}^{2}$ & $\omega_{i}$ & $\sigma_{i, 0}^{2}$ & $k_{x}$ & $\theta_{x}$ & $\omega_{x}$ & $\rho_{0}$ & \\
\hline $\mathrm{i}=1$ & 0.35 & 0.636 & 0.296 & 0.64 & 0.03 & 0.64 & 0.118 & 0.5 & \\
\hline $\mathrm{i}=2$ & 0.35 & 0.636 & 0.296 & 0.16 & & & & & \\
\hline
\end{tabular}

Model 1 is a Brownian motion with constant parameters:

$$
d X_{i t}=\sigma_{i} d W_{i t}
$$

for $i=1,2$, and where $W_{i t}$ are Brownian motions (also denoted by $B_{i t}$ in the next models) and $<d W_{1 t}, d W_{2 t}>=\rho d t$. The initial $\log$ prices are $X_{1,0}=\log (100)$ and $X_{2,0}=\log (40)$.

\footnotetext{
${ }^{9}$ We show results for RK for the two cases with noise.
} 
Model 2 is the Heston model, in which correlations remain constant while volatilities change over time and display jumps, as in Aït-Sahalia et al. (2010) and Shephard and Xiu (2012). For $i=1,2$, we simulate log-prices as

$$
\begin{aligned}
d X_{i t} & =\mu_{i} d t+\sigma_{i t} d W_{i t} \\
d \sigma_{i t}^{2} & =\kappa_{i}\left(\bar{\sigma}_{i}^{2}-\sigma_{i t}^{2}\right) d t+s_{i} \sigma_{i t} d B_{i t}+\sigma_{i t-} J_{i t}^{V o l} d N_{i t},
\end{aligned}
$$

where $<d W_{i t}, d B_{j t}>=\delta_{i j} \rho_{i} d t\left(\delta_{i j}\right.$ denotes the Kronecker delta), $<d W_{1 t}, d W_{2 t}>=\rho d t$, and $\kappa_{i}>0$. The model is calibrated as in Ait-Sahalia et al. (2010). For each new path we generate a starting value for $\sigma_{i t_{0}}^{2}$ from a Gamma distribution $\Gamma\left(2 \kappa_{i} \bar{\sigma}_{i}^{2} / s_{i}^{2}, s_{i}^{2} / 2 \kappa_{i}\right)$, the jump size $\log J_{i t}^{V o l}$ is distributed like $N\left(\theta_{i}, \eta_{i}\right), N_{i t}$ is a Poisson Process with parameter $\lambda_{i}$, and initial log-prices are set equal to $X_{1,0}=\log (100)$ and $X_{2,0}=\log (40)$.

Model 3 also has stochastic volatility and constant correlation. It follows the model on BarndorffNielsen et al. (2011), used for assessing the finite sample properties of multivariate realized kernels. For $i=1,2$, we simulate log-prices as

$$
\begin{aligned}
d X_{i t} & =\mu_{i} d t+d V_{i t}+d F_{i t} \\
d V_{i t} & =\rho_{i} \sigma_{i t} d B_{i t} \\
d F_{i t} & =\sqrt{1-\rho_{i}^{2}} \sigma_{i t} d W_{t} \\
\sigma_{i t} & =\exp \left(\beta_{i 0}+\beta_{i 1} \zeta_{i t}\right) \\
d \zeta_{i t} & =\alpha_{i} \zeta_{i t} d t+d B_{i t},
\end{aligned}
$$

where $<d W_{t}, d B_{j t}>=0,\left\langle d X_{1 t}, d X_{2 t}>=\sqrt{1-\rho_{1}^{2}} \sqrt{1-\rho_{2}^{2}} d t\right.$. We calibrate the model following Barndorff-Nielsen et al. (2011).

Model 4 is a continuous GARCH diffusion. This is the only model allowing stochastic correlations and volatilities. For $i=1,2$, we simulate $\log$-prices as

$$
\begin{aligned}
d X_{i t} & =\sigma_{i t} d W_{i t} \\
d \sigma_{i t}^{2} & =\kappa_{i}\left(\theta_{i}^{2}-\sigma_{i t}^{2}\right) d t+\omega_{i} \sigma_{i t}^{2} d B_{i t} \\
d x_{t} & =\kappa_{x}\left(\theta_{x}-x_{t}\right) d t+\omega_{x} x_{t} d B_{x t} \\
\rho_{t} & =\left(e^{2 x_{t}}-1\right) /\left(e^{2 x_{t}}+1\right),
\end{aligned}
$$

where $<d W_{i t}, d B_{j t}>=0,<d W_{1 t}, d W_{2 t}>=\rho_{t} d t$. We calibrate the parameters following Voev and Lunde (2007) and Andersen and Bollerslev (1998). 
Jumps, noise, asynchronous trading, and accuracy measure. To allow for co-jumps we simulate three independent compound Poisson processes. The two first correspond to the individual jump activity while the third process is common to both assets. We simulate jump arrivals with Poisson processes, and jumps sizes with i.i.d. log-normally distributed random variables. The expected amount of jumps per day corresponds to the parameter of the Poisson processes and are set to 2 for the individual jump activities and 4 for the common jump process. I.e. assets are expected to jump 6 times per day. Jumps sizes are simulated from i.i.d. $N(0, \zeta)$ where $\zeta=\frac{0.7}{252}$.

We simulate noise as in Barndorff-Nielsen et al. (2008), i.e. we assume that $\eta_{t}^{i} \sim$ i.i.d. $N\left(0, \omega_{i}^{2}\right)$ where $\omega_{i}^{2}=\xi^{2} \sqrt{N^{-1} \sum_{m=1}^{N} \sigma_{m / N}^{i, 4}}$ and $\xi^{2}=0.01$.

Non-synchronous trading is introduced using Bernoulli trials. This technique, based on AïtSahalia et al. (2010), selects randomly prices from a grid of evenly spaced transactions. One difference in our scheme is that we simulate probability levels from a uniform distribution over $[0.25 ; 1]$. It enables to cover various regimes of relative liquidity between assets and to test the goodness of our estimators independently from a fixed trading intensity.

Last, to compare the finite sample performances of the estimators we use the root mean squared relative errors (as in Boudt et al. (2011b)):

$$
\operatorname{RMSE}=\sqrt{\frac{1}{T k} \sum_{t=1}^{T}\left\|\operatorname{vech}\left(\operatorname{Estim}_{t}-\mathbf{I C}_{t}\right) \cdot / \operatorname{vech}\left(\mathbf{I C}_{t}\right)\right\|^{2}}
$$

where $\mathbf{E s t i m}_{t}$ denotes the estimator of the integrated covariance matrix for period $t, \mathbf{I C}_{t}$ stands for the integrated covariance matrix for period $t$, vech denotes the vector containing the $k$ is the number of lower diagonal elements of the inputted matrix, $T$ is the amount of simulated periods (days), ./ denotes the element wise division, and $\|$.$\| is the Euclidian norm.$

\subsection{Synchronous prices \& no noise}

Table 2 reports the results. It is divided in four panels, corresponding to different intraday sampling frequencies (30 seconds, and 1, 5, and 15 minutes). Each panel shows the RMSE for eight estimators. The first four are the competitors (RC, BPC, TC and OWC) while the last four are our combinations. For instance MedRV-Spear stands for the MedRV estimator for the volatilities and Spearman's $\rho$ for the correlations. Column wise, the table is divided in the four models, with and without jumps. In the interest of space the QRV estimators are not shown, as MedRV is uniformly better (results are nevertheless reported in Table 7 of the Appendix).

Four are the main findings. First, not surprisingly, RC performs well without jumps but it is very sensitive to them at high frequencies. The other estimators provide better performance in presence of jumps, as they are robust to them. Second, in general the quality of the estimators 
decreases with the sampling frequency. Lower frequencies provide less precise estimates. On average, the relative accuracy of the estimators for 1-, 5- and 15-minute returns compared to the RMSE of estimators computed on basis of 30-second returns are of order close to $\sqrt{2}, \sqrt{10}$ and $\sqrt{30}$ respectively. Similar results are found by Boudt et al. (2011b). In fact, our model 3 corresponds to their main model, except that the jump process is different.

Third, the threshold used for TC provides good results, as the performances of TC are close to those of RC in absence of jumps and far better when jumps are added. ${ }^{10}$ Moreover, most of the time OWC outperforms the other estimators, followed by TC for high frequencies. Our combinations are generally less accurate than these two estimators and perform on average better than the BPC. The goodness of our estimators will appear more clearly in case of asynchronous trading.

Fourth, and as briefly mentioned above, combinations based on median operators for volatility measures provide in general more accurate measures than those based on quantiles. Moreover, quadrant correlations perform in a less convincing way than Gaussian ranks, Spearman's $\rho$ and Kendall's $\tau$. In the sequel we only show results for the combination based on median measures for volatilities and Spearman's $\rho$ and Gaussian ranks for correlations. We skip Kendall's $\tau$ as it is less computationally efficient that the others.

\subsection{Asynchronous prices \& no noise}

We study the impact of asynchronous trading. Following Mancini and Gobbi (2012), RC and TC are implemented with the pseudo-aggregation scheme proposed by Hayashi and Yoshida (2005); we denote this estimator as HY-RC and HY-TC. For OWC and BPC, we follow Boudt et al. (2011b) and use returns aligned on a 5-minute grid with previous-tick interpolation in order to avoid microstructure effects related to asynchronous trading; we denote the estimators PT-BPC and PT-OWC. Our estimators are computed with data synchronized using refresh time (denoted RT), 30-seconds previous tick (denoted PT) and 30-seconds linear interpolation (denoted LI).

Results are in Table 3. We draw two conclusions. First, estimators based on the HayashiYoshida scheme are very good. The technique provides an efficient way to cumulate overlapping log-returns. HY-TC is the most efficient way to estimate the integrated covariance matrix. LTOWC losses efficiency because of the lower sampling frequency. Nevertheless, at higher frequencies, biases related with the Epps effect appear, as pointed out in Boudt et al. (2011b).

Second, our estimators perform on average less efficiently than HY-TC. The 30-second linear interpolation scheme leads to the best results among the different combinations for disentangled realized covariances. Without jumps it performs less efficiently than HY-RC but better than other estimators. In presence of jumps it performs better than all its competitors except for

\footnotetext{
${ }^{10}$ Note that TC is a truncated version of $\mathrm{RC}$ and should provide equivalent results in absence of jumps
} 
Table 2: Monte Carlo - Synchronous trading and no noise

\begin{tabular}{|c|c|c|c|c|c|c|c|c|}
\hline 30-second retur & \multicolumn{2}{|c|}{$\begin{array}{c}\text { Model } 1 \\
\text { no jumps jumps }\end{array}$} & \multicolumn{2}{|c|}{$\begin{array}{c}\text { Model } 2 \\
\text { no jumps jumps }\end{array}$} & \multicolumn{2}{|c|}{$\begin{array}{c}\text { Model } 3 \\
\text { no jumps jumps }\end{array}$} & \multicolumn{2}{|c|}{$\begin{array}{c}\text { Model } 4 \\
\text { no jumps jumps }\end{array}$} \\
\hline $\mathrm{RC}$ & 0.0819 & 24.0512 & 0.9457 & 9.4206 & 0.0542 & 3.9498 & 0.0622 & 3.3527 \\
\hline $\mathrm{BPC}$ & 0.1004 & 1.6216 & 0.9431 & 1.5826 & 0.0622 & 0.3409 & 0.0729 & 0.3994 \\
\hline $\mathrm{TC}$ & 0.0864 & 0.0849 & 0.9178 & 0.961 & 0.0602 & 0.0578 & 0.0675 & 0.0648 \\
\hline OWC & 0.0843 & 0.0865 & 0.936 & 0.9697 & 0.0561 & 0.0563 & 0.0643 & 0.0647 \\
\hline Med-Ken & 0.105 & 0.1128 & 0.9546 & 1.0082 & 0.0826 & 0.0844 & 0.0897 & 0.0919 \\
\hline Med-Spear & 0.1051 & 0.1142 & 0.9539 & 1.0067 & 0.0829 & 0.0844 & 0.0899 & 0.0915 \\
\hline Med-Quad & 0.1308 & 0.1343 & 0.9549 & 1.0083 & 0.0866 & 0.0879 & 0.0999 & 0.1007 \\
\hline Med-Gauss & 0.1026 & 0.1372 & 0.9525 & 1.0066 & 0.082 & 0.0834 & 0.0883 & 0.0905 \\
\hline \multicolumn{9}{|c|}{ 1-minute returns } \\
\hline $\mathrm{RC}$ & 0.1161 & 24.0367 & 0.9539 & 9.4143 & 0.0761 & 3.9549 & 0.0889 & 3.3524 \\
\hline $\mathrm{BPC}$ & 0.1403 & 2.2101 & 0.9528 & 1.9298 & 0.0871 & 0.4947 & 0.1027 & 0.556 \\
\hline $\mathrm{TC}$ & 0.1203 & 0.1233 & 0.9236 & 0.9723 & 0.0832 & 0.0831 & 0.0938 & 0.0917 \\
\hline OWC & 0.1199 & 0.1207 & 0.9425 & 0.9772 & 0.0792 & 0.0806 & 0.0918 & 0.0916 \\
\hline Med-Ken & 0.1496 & 0.1694 & 0.9567 & 1.04 & 0.1165 & 0.1222 & 0.1247 & 0.1329 \\
\hline Med-Spear & 0.1495 & 0.1726 & 0.9552 & 1.037 & 0.1169 & 0.1219 & 0.125 & 0.1318 \\
\hline Med-Quad & 0.1837 & 0.1933 & 0.9585 & 1.0386 & 0.1215 & 0.1267 & 0.1384 & 0.1458 \\
\hline Med-Gauss & 0.1461 & 0.2102 & 0.9526 & 1.0364 & 0.116 & 0.1208 & 0.1233 & 0.1306 \\
\hline \multicolumn{9}{|c|}{ 5-minute returns } \\
\hline $\mathrm{RC}$ & 0.2579 & 24.092 & 0.9735 & 9.473 & 0.1677 & 3.9575 & 0.1971 & 3.3675 \\
\hline $\mathrm{BPC}$ & 0.3092 & 5.4849 & 0.9697 & 3.3473 & 0.1944 & 1.1282 & 0.2258 & 1.1754 \\
\hline $\mathrm{TC}$ & 0.263 & 0.9183 & 0.9436 & 1.3712 & 0.1788 & 0.3001 & 0.2053 & 0.344 \\
\hline OWC & 0.2693 & 0.2931 & 0.951 & 1.1137 & 0.1774 & 0.2116 & 0.2055 & 0.2399 \\
\hline Med-Ken & 0.3404 & 0.5774 & 1.0411 & 1.3723 & 0.2528 & 0.3308 & 0.2769 & 0.3825 \\
\hline Med-Spear & 0.3342 & 0.582 & 1.0345 & 1.356 & 0.2529 & 0.3256 & 0.2751 & 0.3708 \\
\hline Med-Quad & 0.4015 & 0.5564 & 1.0443 & 1.3598 & 0.2641 & 0.3333 & 0.3045 & 0.3901 \\
\hline Med-Gauss & 0.328 & 0.6369 & 1.0289 & 1.352 & 0.2519 & 0.3229 & 0.2737 & 0.3667 \\
\hline \multicolumn{9}{|c|}{ 15-minute returns } \\
\hline $\mathrm{RC}$ & 0.4426 & 24.0955 & 1.0246 & 9.3805 & 0.2914 & 3.9825 & 0.3345 & 3.4213 \\
\hline $\mathrm{BPC}$ & 0.5215 & 9.391 & 1.0099 & 4.83 & 0.3334 & 1.7732 & 0.3801 & 1.7971 \\
\hline $\mathrm{TC}$ & 0.4485 & 5.8392 & 0.9923 & 3.5511 & 0.3104 & 1.2296 & 0.3495 & 1.3368 \\
\hline OWC & 0.4794 & 2.9862 & 0.9718 & 2.4035 & 0.3268 & 0.7733 & 0.3709 & 0.866 \\
\hline Med-Ken & 0.5908 & 1.5248 & 1.2072 & 2.3365 & 0.4522 & 0.7293 & 0.4835 & 0.8947 \\
\hline Med-Spear & 0.5824 & 1.5038 & 1.2025 & 2.3021 & 0.4514 & 0.7205 & 0.481 & 0.8744 \\
\hline Med-Quad & 0.6884 & 1.3666 & 1.2375 & 2.323 & 0.467 & 0.7429 & 0.5233 & 0.9166 \\
\hline Med-Gauss & 0.5714 & 1.6556 & 1.1929 & 2.3042 & 0.447 & 0.7135 & 0.4736 & 0.8711 \\
\hline
\end{tabular}

Monte Carlo simulation results for eight estimators of the integrated covariance matrix under four different models specified with and without jumps. The columns report the root mean square errors (RMSE) computed as in (17) for 10000 draws of 23400 observations recorded over one period of trading (6.5 hours). Prices are simulated simultaneously each second and without noise. Every panel of the table contains the four competitors (RC, BPC, TC and OWC), followed by the disentangled estimators, which are computed with 5 blocks (except for 15-minute returns for which only 1 block is used).

HY-TC. This result, though unfavorable for the class of disentangled realized measures, is logical. Indeed, Hayashi-Yoshida scheme uses all the data points by aggregating returns which have been 
recorded on overlapping time periods. As mentioned previously, interpolation techniques project prices on fixed grids and inevitably delete data points, just as refresh time and other generalized synchronization schemes.

Table 3: Monte Carlo - Asynchronous trading and no noise

\begin{tabular}{ccccccccc}
\hline & \multicolumn{3}{c}{ Model 1 } & \multicolumn{2}{c}{ Model 2} & \multicolumn{2}{c}{ Model 3 } & \multicolumn{2}{c}{ Model 4} \\
& no jumps jumps & no jumps jumps & no jumps jumps & no jumps jumps \\
\hline HY-RC & 0.0299 & 17.0792 & 0.9344 & 6.8738 & 0.018 & 2.7555 & 0.0213 & 2.329 \\
PT-BPC & 0.3173 & 4.0546 & 0.9711 & 2.7144 & 0.191 & 0.8133 & 0.225 & 0.9235 \\
HY-TC & 0.0395 & 0.0344 & 0.9046 & 0.9257 & 0.0313 & 0.0285 & 0.0337 & 0.0303 \\
PT-OWC & 0.274 & 0.2915 & 0.9482 & 1.1003 & 0.176 & 0.2113 & 0.2022 & 0.247 \\
PT-Spear & 0.078 & 0.1006 & 0.9265 & 0.9452 & 0.0392 & 0.0411 & 0.0509 & 0.0491 \\
LI-Spear & 0.0723 & 0.0919 & 0.9315 & 0.9502 & 0.0279 & 0.0295 & 0.0419 & 0.0415 \\
RT-Spear & 0.0661 & 0.0684 & 0.9203 & 0.9381 & 0.0623 & 0.0624 & 0.064 & 0.0619 \\
PT-Gauss & 0.0759 & 0.136 & 0.9254 & 0.9455 & 0.0404 & 0.0435 & 0.051 & 0.0477 \\
LI-Gauss & 0.0695 & 0.1277 & 0.9303 & 0.9506 & 0.0283 & 0.0313 & 0.0411 & 0.04 \\
RT-Gauss & 0.0661 & 0.0749 & 0.9201 & 0.9381 & 0.0632 & 0.0635 & 0.0644 & 0.0618 \\
\hline
\end{tabular}

\subsection{Synchronous prices \& noise}

We now study the impact of noise with synchronous trading. Our estimators are computed with sparse sampling (denoted by B at the end of the name; e.g. SpearB). sub-sampling (denoted by $\mathrm{S}$ ), and pre-averaging (denoted by $\mathrm{P}$ ). We add to the comparison the realized kernels of BarndorffNielsen et al. (2011) (denoted RK) and we skip results for 30 seconds, as it is well known to be a too high frequency in the presence of noise (i.e. upward biases in th realized masures). Results are in Table 4 and three are the main conclusions.

First, pre-averaging is the most efficient technique for dealing with microstructure noise, while sparse sampling displays the worse results. Sub-sampling - implemented on 5-minute returns increases the efficiency of the estimates compared to sparse sampling, but provides higher RMSE than pre-averaged estimators. However, as the sampling frequency decreases from 1 to 5 minute, DRC estimators based on blocks become more efficient, revealing the goodness of a sparser grid of time coordinates when prices are noisy. Additionally, results for 15 minutes-returns provide less clear cut results and advocates the use of 5 minute returns when using sparse sampling. 
Table 4: Monte Carlo study - Synchronous trading

\begin{tabular}{|c|c|c|c|c|c|c|c|c|}
\hline 1-minute returns & \multicolumn{2}{|c|}{$\begin{array}{c}\text { Model } 1 \\
\text { no jumps jumps }\end{array}$} & \multicolumn{2}{|c|}{$\begin{array}{c}\text { Model } 2 \\
\text { no jumps jumps }\end{array}$} & \multicolumn{2}{|c|}{$\begin{array}{c}\text { Model } 3 \\
\text { no jumps jumps }\end{array}$} & \multicolumn{2}{|c|}{$\begin{array}{c}\text { Model } 4 \\
\text { no jumps jumps }\end{array}$} \\
\hline $\mathrm{RC}$ & 0.669 & 24.62 & 1.352 & 9.941 & 0.647 & 4.26 & 0.653 & 3.758 \\
\hline $\mathrm{BPC}$ & 0.717 & 2.978 & 1.369 & 2.474 & 0.683 & 1.095 & 0.691 & 1.178 \\
\hline $\mathrm{TC}$ & 0.648 & 0.668 & 1.314 & 1.347 & 0.626 & 0.654 & 0.632 & 0.655 \\
\hline OWC & 0.661 & 0.666 & 1.337 & 1.355 & 0.638 & 0.656 & 0.644 & 0.658 \\
\hline SpearB & 0.689 & 0.747 & 1.361 & 1.443 & 0.664 & 0.711 & 0.67 & 0.717 \\
\hline GaussB & 0.686 & 0.769 & 1.359 & 1.447 & 0.663 & 0.71 & 0.669 & 0.717 \\
\hline SpearS & 0.261 & 0.561 & 1.031 & 1.332 & 0.203 & 0.327 & 0.219 & 0.374 \\
\hline GaussS & 0.257 & 0.739 & 1.029 & 1.344 & 0.202 & 0.327 & 0.217 & 0.384 \\
\hline SpearP & 0.141 & 0.201 & 0.981 & 1.076 & 0.105 & 0.136 & 0.116 & 0.15 \\
\hline GaussP & 0.137 & 0.286 & 0.979 & 1.079 & 0.103 & 0.134 & 0.114 & 0.152 \\
\hline RK & 0.115 & 24.337 & 0.951 & 9.612 & 0.076 & 3.977 & 0.088 & 3.383 \\
\hline \multicolumn{9}{|c|}{ 5-minute returns } \\
\hline $\mathrm{RC}$ & 0.323 & 24.423 & 1.033 & 9.659 & 0.224 & 3.931 & 0.251 & 3.462 \\
\hline $\mathrm{BPC}$ & 0.378 & 5.636 & 1.028 & 3.397 & 0.244 & 1.193 & 0.278 & 1.297 \\
\hline $\mathrm{TC}$ & 0.319 & 1.011 & 0.997 & 1.401 & 0.222 & 0.406 & 0.249 & 0.442 \\
\hline OWC & 0.329 & 0.368 & 1.009 & 1.141 & 0.223 & 0.297 & 0.252 & 0.325 \\
\hline SpearB & 0.411 & 0.709 & 1.106 & 1.425 & 0.315 & 0.439 & 0.343 & 0.487 \\
\hline GaussB & 0.404 & 0.764 & 1.1 & 1.423 & 0.314 & 0.437 & 0.34 & 0.485 \\
\hline SpearS & 0.261 & 0.561 & 1.031 & 1.332 & 0.203 & 0.327 & 0.219 & 0.374 \\
\hline GaussS & 0.257 & 0.739 & 1.029 & 1.344 & 0.202 & 0.327 & 0.217 & 0.384 \\
\hline SpearP & 0.141 & 0.201 & 0.981 & 1.076 & 0.105 & 0.136 & 0.116 & 0.15 \\
\hline GaussP & 0.137 & 0.286 & 0.979 & 1.079 & 0.103 & 0.134 & 0.114 & 0.152 \\
\hline RK & 0.115 & 24.337 & 0.951 & 9.612 & 0.076 & 3.977 & 0.088 & 3.383 \\
\hline \multicolumn{9}{|c|}{ 15-minute returns } \\
\hline $\mathrm{RC}$ & 0.465 & 24.269 & 1.055 & 9.658 & 0.303 & 3.938 & 0.35 & 3.454 \\
\hline $\mathrm{BPC}$ & 0.553 & 9.85 & 1.036 & 5.07 & 0.341 & 1.844 & 0.4 & 1.891 \\
\hline $\mathrm{TC}$ & 0.468 & 6.195 & 1.021 & 3.758 & 0.319 & 1.406 & 0.363 & 1.401 \\
\hline OWC & 0.501 & 3.273 & 0.997 & 2.402 & 0.331 & 0.802 & 0.382 & 0.906 \\
\hline SpearB & 0.61 & 1.579 & 1.248 & 2.279 & 0.466 & 0.784 & 0.505 & 0.928 \\
\hline GaussB & 0.596 & 1.733 & 1.24 & 2.283 & 0.462 & 0.777 & 0.497 & 0.926 \\
\hline SpearS & 0.261 & 0.561 & 1.031 & 1.332 & 0.203 & 0.327 & 0.219 & 0.374 \\
\hline GaussS & 0.257 & 0.739 & 1.029 & 1.344 & 0.202 & 0.327 & 0.217 & 0.384 \\
\hline SpearP & 0.141 & 0.201 & 0.981 & 1.076 & 0.105 & 0.136 & 0.116 & 0.15 \\
\hline GaussP & 0.137 & 0.286 & 0.979 & 1.079 & 0.103 & 0.134 & 0.114 & 0.152 \\
\hline RK & 0.115 & 24.337 & 0.951 & 9.612 & 0.076 & 3.977 & 0.088 & 3.383 \\
\hline
\end{tabular}

Monte Carlo simulation results for eight estimators of the integrated covariance matrix under four different models specified with and without jumps. The columns report the root mean square errors (RMSE) computed as in (17) for 10000 draws of 23400 observations recorded over one period of trading (6.5 hours). Prices are simulated simultaneously each second and with noise. The abbreviations correspond to the the name of the estimator followed by the noise reduction technique $(\mathrm{B}=$ sparse sampling, $\mathrm{S}=$ sub-sampling, and $\mathrm{P}=$ pre-averaging). Disentangled estimators are computed with 5 blocks, except for 15-minute returns for which only 1 block is used. 
Second, for all approaches, the DRC estimator implemented with Gaussian ranks and Spearman's $\rho$ provide similar precision, except for model 1 for which Spearman's $\rho$ has smaller RMSE. These results, jointly with the milder condition for positive definiteness, supports the use of Gaussian ranks.

Third, when jumps are added, the pre-averaged DRC estimators provide uniformly more precise estimates than the competitors. However, in absence of jumps, realized kernels provide slightly more precise results. For 5 -minute returns, the two subsampled combinations perform better than most of the competitors except for OWC. Depending on the simulated model, one performs better than the other. The competing estimators are described by their respective authors as the least sensitive to noise when computed on basis of 5-minute returns. This statement is verified: the RMSE's are on average smaller for the middle panel.

\subsection{Asynchronous prices \& noise}

Last, we analyze the goodness of the estimators with asynchronous and noisy observations. For the competing estimators, we focus on 5-minutes frequency, as previous sub-sections showed that this frequency gave the best results. Likewise, the DRC estimators are shown using pre-averaging and subsampling, since sparse sampling gave the worst results in previous subsections. ${ }^{11}$

We synchronize using last tick interpolation at a frequency depending on the trading intensity of the considered securities. ${ }^{12}$ Note that Hayashi and Yoshida was used for the RC and TC estimators when data are clean of noise. However, if returns are noisy, HY becomes impracticable and hence the authors advise to use last tick interpolation. Realized kernels are implemented following Barndorff-Nielsen et al. (2011) with refresh time and subsampled realized variances to compute to optimal bandwidth.

Results are shown in Table 5. As in the case of synchronous trading and noise, pre-averaging provides better estimates than subsampling. In the absence of jumps, realized kernels provide the best results, followed by the pre-averaged DRC. With jumps however the realized kernels provide inaccurate measures. They are as sensitive as the baseline realized covariances to jumps.

Pre-averaged DRC strike hence a good balance between jump-robustness and precision and form a serious alternative to other realized measures. Additionally, results are similar for DRC based on Spearman rho and Gaussian ranks. When jumps occur, they perform slightly less efficiently than OWC. Nevertheless, this drawback is compensated by a less demanding computational effort.

\footnotetext{
${ }^{11}$ Pre-averaging is implemented on basis of the new grid of synchronous prices and subsampling is conducted with 5-minute returns also sampled from the new grid.

${ }^{12}$ We compute the $75 \%$ quantile of time intervals between trades for each asset, take the minimum and stamp the sampling frequency to the nearest second. The $75 \%$ quantile may be considered as a conservative choice but it allows to keep under control effects of asynchronous trading causing downward biases in correlations.
} 
Table 5: Monte Carlo study - Asynchronous trading and noise

\begin{tabular}{ccccccccc}
\hline \hline & \multicolumn{2}{c}{$\begin{array}{c}\text { Model 1 } \\
\text { no jumps jumps }\end{array}$} & \multicolumn{2}{c}{$\begin{array}{c}\text { Model 2 } \\
\text { no jumps jumps }\end{array}$} & \multicolumn{2}{c}{$\begin{array}{c}\text { Model } 3 \\
\text { no jumps jumps }\end{array}$} & no jumps jumps \\
& & & & & & & & \\
& & & & & & & & \\
\hline LT-RC & 0.404 & 17.47 & 4.625 & 9.318 & 0.386 & 2.965 & 0.264 & 2.439 \\
LT-BPC & 0.455 & 4.366 & 5.082 & 6.808 & 0.407 & 1.119 & 0.291 & 1.015 \\
LT-TC & 0.395 & 0.912 & 4.567 & 5.413 & 0.374 & 0.56 & 0.26 & 0.424 \\
LT-OWC & 0.404 & 0.455 & 4.55 & 4.897 & 0.376 & 0.477 & 0.264 & 0.331 \\
LT-SpearS & 0.344 & 0.65 & 4.599 & 5.226 & 0.355 & 0.495 & 0.233 & 0.365 \\
LT-GaussS & 0.341 & 0.82 & 4.598 & 5.255 & 0.355 & 0.496 & 0.231 & 0.374 \\
LT-SpearP & 0.184 & 0.295 & 1.366 & 1.527 & 0.145 & 0.191 & 0.144 & 0.191 \\
LT-GaussP & 0.18 & 0.454 & 1.365 & 1.55 & 0.143 & 0.191 & 0.141 & 0.199 \\
RK & 0.154 & 17.434 & 1.030 & 7.030 & 0.112 & 2.827 & 0.108 & 2.387 \\
\hline \hline
\end{tabular}

Monte Carlo simulation results for six estimators of the integrated covariance matrix under four different models specified with and without jumps. The columns report the root mean square errors (RMSE) computed as in (17) for 10000 draws of 23400 observations recorded over one period of trading (6.5 hours). Prices are simulated asynchronously and with noise. The abbreviations correspond to the used synchronization technique, followed by the name of the estimator, and the noise reduction technique. Disentangled estimators are sub-sampled.

\section{Empirical application}

We now study the gains of our estimators from a financial perspective. We proceed with a longshort portfolio management exercise using 52 large stocks traded on the NYSE from 2006 to 2012.

Volatility timing strategies are based on conditional covariance matrix of daily returns. Since realized covariances are ex-post measures of the co-variation between assets, we use one-day ahead forecasts of the covariance matrix $\boldsymbol{\Sigma}_{t}$ in the portfolio construction. The investor updates and rebalances his portfolio every day on basis of the new information generated by markets. If we consider a market composed of $p$ assets that the investor can select in his portfolio, the optimal $p \times 1$ vector of weights $\boldsymbol{\omega}_{t}$ for the portfolio allocation are computed by solving standard conditional mean-variance criterion:

$$
\min _{\boldsymbol{\omega}_{t}}\left(\boldsymbol{\omega}_{t} \boldsymbol{\Sigma}_{t} \boldsymbol{\omega}_{t}\right)
$$

subject to $\boldsymbol{\omega}_{t}^{\prime} \mathbf{1}=1$. The solution $\boldsymbol{\omega}_{t}$ to this strategy is well known:

$$
\boldsymbol{\omega}_{t}=\frac{\boldsymbol{\Sigma}_{t}^{-1} \mathbf{1}}{\mathbf{1}^{\prime} \boldsymbol{\Sigma}_{t}^{-1} \mathbf{1}}
$$

We evaluate the performance of the portfolio on basis of five criteria: i) the annualized mean returns, ii) the annualized standard deviation, iii) the annualized Sharp ratio, iv) the annualized average turnover (given by $T O_{t}=\left|\boldsymbol{\omega}_{t}-\boldsymbol{\omega}_{t-1}\right| \mathbf{1}_{p}$ ), and $\mathrm{v}$ ) the cumulated performance in terms of the return on investment. 


\subsection{Models for conditional covariance matrices}

The practical implementation of volatility timing strategies require conditional covariance matrix measurement, i.e. the covariance matrix at time $t+1$ given information up to time $t$. We consider a set of $p$ stocks and denote the daily returns at $t$ by $\mathbf{r}_{t}$ and realized covariance measures on day $t$ as $\mathbf{V}_{t}$. Investors believe that asset returns behave as:

$$
\mathbf{r}_{t}=\boldsymbol{\mu}_{t}+\boldsymbol{\Sigma}_{t}^{\frac{1}{2}} \mathbf{z}_{t}
$$

where $\mathbf{z}_{t} \sim$ i.i.d. $N\left(0, \mathbf{I}_{p}\right)$ and $\mathbf{I}_{p}$ denotes the identity matrix of size $p$. Moreover, $\boldsymbol{\mu}_{t}=E\left[\mathbf{r}_{t} \mid \mathcal{A}_{t-1}\right]$ and $\boldsymbol{\Sigma}_{t}=V\left[\mathbf{r}_{t} \mid \mathcal{A}_{t-1}\right]$. We assume $\boldsymbol{\mu}_{t}$ to be constant and estimate it with the sample mean. Three models are considered for $\boldsymbol{\Sigma}_{t}$. Two of them rely on realized covariances and one on daily returns only. In other words, we compare investments conducted on basis of two different information sets: $\mathcal{A}_{t}^{L F}$ and $\mathcal{A}_{t}^{H F} . \mathcal{A}_{t}^{L F}$ denotes the low frequency information set generated by daily returns and $\mathcal{A}_{t}^{H F}$ is the high frequency information set generated by daily returns and realized covariances. More rigorously we have: $\mathcal{A}_{t}^{L F}=\sigma\left(\chi_{s}, s \leq t\right)$ where $\chi_{s}=\left\{\mathbf{r}_{s}\right\}$, and $\mathcal{A}_{t}^{H F}=\sigma\left(\chi_{s}, s \leq t\right)$ where $\chi_{s}=\left\{\mathbf{r}_{s}, V_{s}\right\}$.

The low-frequency benchmark model is the DCC of Engle (2002) (DCC):

$$
\begin{aligned}
& \boldsymbol{\Sigma}_{t}=\mathbf{D}_{t} \mathbf{R}_{t} \mathbf{D}_{t} \text { where } \\
& \mathbf{R}_{t}=\left(\mathbf{Q}_{t} \odot \mathbf{1}_{p}\right)^{-1 / 2} \mathbf{Q}_{t}\left(\mathbf{Q}_{t} \odot \mathbf{1}_{p}\right)^{-1 / 2}, \\
& \mathbf{Q}_{t}=(1-\alpha-\beta) \overline{\mathbf{Q}}+\alpha \mathbf{u}_{t-1} \mathbf{u}_{t-1}^{\prime}+\beta \mathbf{Q}_{t-1},
\end{aligned}
$$

$\mathbf{D}_{t}=\left(\boldsymbol{\Sigma}_{t} \odot \mathbf{I}_{p}\right)^{1 / 2}, u_{i, t}=z_{i, t} / h_{i, t}^{1 / 2}$, and $h_{i, t}^{1 / 2}$ denotes the elements on the diagonal of $\mathbf{D}_{t}$, i.e. the univariate volatilities which are specified as $\operatorname{GARCH}(1,1)$ processes. Long memory is not considered since only one step ahead forecasts are required.

We consider two models for the conditional covariance matrix based on high-frequency data. First, we estimate a rolling window volatility model. ${ }^{13}$ The estimation procedure closely follows De Pooter et al. (2008) and Fleming et al. (2003). This technique is based on the work of Foster and Nelson (1996) and Andreou and Ghysels (2002). The daily conditional covariance matrix based on high-frequency data is:

$$
\boldsymbol{\Sigma}_{t}=\exp (-\alpha) \boldsymbol{\Sigma}_{t-1}+\alpha \exp (-\alpha) \mathbf{V}_{t-1}
$$

Smaller values for the decay parameter $\alpha$ point to a less informative innovation process $V_{t-1}$, i.e. the estimator is too noisy from a portfolio construction perspective, while a large value for $\alpha$ point to more informative innovations (Bannouh et al. (2009)). Fleming et al. (2003) and De Pooter

\footnotetext{
13 "Rolling window" is the name of the model, which is different to rolling unconditional estimation by moving a window of observations.
} 
et al. (2008) point out that statistically optimal parameters do not lead to optimal financial performances. Indeed, estimating $\alpha$ via maximum likelihood does not provide the portfolio with the best risk-return trade-off. This is why Boudt et al. (2012) consider two optimality criteria: maximum likelihood and minimum volatility of the investment. Yet, to be fair with the other two models, we use maximum likelihood.

The second model with high frequency data is the HEAVY of Noureldin et al. (2012). Their model is specified as the BEKK of Engle and Kroner (1995) but lagged values of the cross products of returns which are replaced by lagged values of the realized covariances:

$$
\boldsymbol{\Sigma}_{t}=\mathbf{\Omega}+\mathbf{B} \boldsymbol{\Sigma}_{t-1} \mathbf{B}^{\prime}+\mathbf{A} \mathbf{V}_{t-1} \mathbf{A}^{\prime}
$$

Realized covariances are modeled as $E\left[\mathbf{V}_{t} \mid \mathcal{A}_{t-1}^{H F}\right]=\mathbf{M}_{t}$ for which $\mathbf{M}_{t}$ is again specified as a BEKK:

$$
\mathbf{M}_{t}=\mathbf{\Omega}_{M}+\mathbf{D M}_{t-1} \mathbf{D}^{\prime}+\mathbf{G V}_{t-1} \mathbf{G}^{\prime}
$$

Various specifications can be adopted to limit the amount of parameters. We study the scalarBEKK specification whose properties are illustrated in the empirical study of Noureldin et al. (2012). The Wishart distribution is assumed and estimation is done by maximum likelihood.

\section{$6.2 \quad$ Results}

The investment universe is composed of 52 large stocks traded on the NYSE. Data consists of trades and prices. ${ }^{14}$ The data covers the period from October 2006 to April 2012 for a total of 1403 observations. The database is cleaned as in Barndorff-Nielsen et al. (2009).

Results of the minimum variance portfolio are in Table 6 and Figure 3. This table summarizes the performance for portfolios constructed on basis of the rolling window model, the HEAVY model and the $D C C_{E}$. Results for the $D C C_{E}$ are reported under the results of the HEAVY model. Mean return (denoted Mean) and standard deviation (denoted Std. dev.) are annualized, while turnover (denoted TO) is daily. The fifth performance measure is displayed in the figure. It is shows the cumulated performance for the same estimators as in the table in terms of return on investment of one monetary unit invested in October 2006. All the performances based on realized measures are located in the shaded area (delimited by the minimum and the maximum daily performances). Other lines illustrate performances on the same period for the strategy based on the $D D C_{E}$ (solid line), and the performance of the Spider S\&P 500 ETF (SPY; dashed). The solid black line, denoted DRC-GR in the figures, represents the performance of the portfolio based on the disentangled realized covariances with Gaussian ranks.

\footnotetext{
${ }^{14}$ Tickers: AA, ABT, AES, AKS, AMD, BMY, BSX, C, CAG, CBS, COH, CSX, CVX, D, DIS, DNR, EMC, EXC, FCX, GE, GIS, GLW, HAL, HPQ, HST, IRM, JCP, JPM, KEY, KO, MO, MRK, MS, NBR, NEM, ORCL, PFE, PG, RF, S, SLB, T, TJX, USB, VLO, VZ, WFC, WMT, WU, WY, XRX, SPY.
} 
Table 6: Minimum variance portfolio - Performance measures

\begin{tabular}{cccccccccc}
\hline \hline Model & \multicolumn{4}{c}{ Rolling Window } & \multicolumn{5}{c}{ HEAVY } \\
Performance measure & Mean & Sdt dev. & Sharp & TO & $\alpha$ & Mean & Sdt dev. & Sharp & TO \\
\hline RC & $9.25 \%$ & $12.63 \%$ & 0.732 & 0.12 & 0.037 & $12.16 \%$ & $12.18 \%$ & 0.998 & 0.29 \\
BPC & $7.83 \%$ & $12.99 \%$ & 0.603 & 0.11 & 0.034 & $12.64 \%$ & $12.24 \%$ & 1.032 & 0.24 \\
TC & $7.97 \%$ & $12.93 \%$ & 0.616 & 0.09 & 0.030 & $12.84 \%$ & $12.24 \%$ & 1.049 & 0.34 \\
OWC & $9.36 \%$ & $13.04 \%$ & 0.718 & 0.09 & 0.029 & $12.05 \%$ & $12.31 \%$ & 0.979 & 0.36 \\
RK & $8.19 \%$ & $12.86 \%$ & 0.637 & 0.11 & 0.036 & $9.79 \%$ & $12.38 \%$ & 0.791 & 0.36 \\
SpearS & $6.83 \%$ & $13.44 \%$ & 0.508 & 0.07 & 0.024 & $13.17 \%$ & $12.43 \%$ & 1.059 & 0.51 \\
GaussS & $6.35 \%$ & $13.42 \%$ & 0.473 & 0.07 & 0.024 & $13.33 \%$ & $12.44 \%$ & 1.072 & 0.53 \\
SpearP & $7.59 \%$ & $13.50 \%$ & 0.562 & 0.07 & 0.020 & $12.82 \%$ & $12.48 \%$ & 1.028 & 0.42 \\
GaussP & $6.44 \%$ & $13.55 \%$ & 0.475 & 0.07 & 0.020 & $12.97 \%$ & $12.49 \%$ & 1.038 & 0.43 \\
\hline$D C C_{E}$ & - & - & - & - & - & $6.59 \%$ & $13.38 \%$ & 0.493 & 0.54 \\
\hline \hline
\end{tabular}

This table summarizes the performance for portfolios constructed on basis of the rolling window model, the HEAVY model and the $D C C_{E}$. Results for the $D C C_{E}$ are reported under the results of the HEAVY model. Standard deviation and mean return have been annualized while turnover has been kept on daily basis.

The strategy based on the HEAVY model is more profitable (mean returns) than based on the rolling window model. It is also less risky (of the order of 12.4\%). Compared with the average S\&P 500 VIX over the sample period (24.66\%), the volatility of the portfolio using the HEAVY model is unusually low. Moreover, on average, the Sharp ratios are higher than for the rolling window model. These results are not surprising since the HEAVY model has a richer specification than the rolling window. Nevertheless, it has to be noted that the portfolios constructed on basis of the HEAVY model are less stable as the turnover is higher. The DCC behaves worse that the other models. The average return is lower while the annualized volatility remain similar to other strategies. This performance may be due to the fact that the $D C C_{E}$ is based on a poorer information set. The return on investment (see figure) confirms that investing on basis of the HEAVY model is preferable to the rolling window and the $D C C_{E}$.

\section{Conclusion}

We study the properties of the class of disentangled estimators of the integrated covariance matrix of Itō semimartingales in an extensive Monte Carlo study. We cover different scenarios when efficient prices are observable or contaminated by noise, with and without jumps, and with synchronous or non-synchronous trading. We show that if one selects the right combinations of estimators and robustification techniques, disentangled realized covariances prove to be as precise as other measures, jump robust, simpler, robust to noise, positive definite, and computationally efficient.

Our main conclusion is that if observations are non-synchronous and noisy (as it is the case 

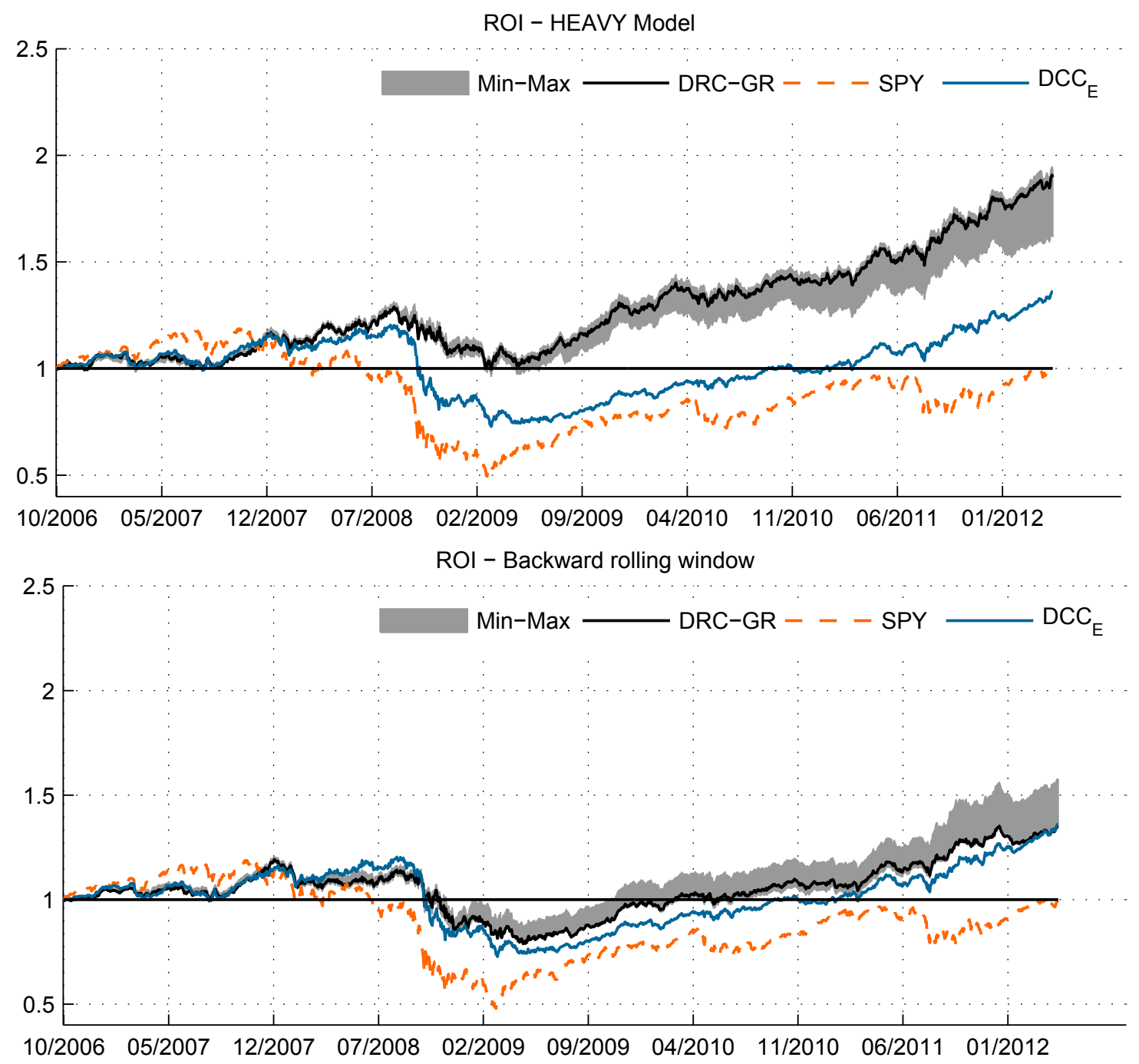

Figure 3: This figure displays the cumulated performance for the different estimators in terms of return on investment of one monetary unit invested in October 2006. All the performances are located in the shaded area delimited by the minimum and the maximum daily performance. Other lines illustrate performances on the same period for the strategy based on the $D D C$ and the performance of a S\&P 500 ETF. The solid black line represents the performance of the portfolio based on the Gaussian ranks disentangled realized covariances.

of multivariate high frequency data), the subsampled version of disentangled estimators based on Gaussian ranks (for the correlations) and median deviations (for the volatilities) is the most appropriate metrics in terms of root mean square error. This result dovetails with Boudt et al. (2012).

Moreover, this finding is confirmed by an empirical analysis based on a cross-section of stocks traded on the NYSE. Performances from a minimum variance portfolio strategy based on our favorite estimator provide the highest mean return, lower volatility, highest Sharp ratio, and 
highest return on investment.

\section{Acknowledgements}

This work was partly done while Harry Vander Elst visited CREATES at Aarhus University and when David Veredas hold the Banco de Santander Chair of Excellence at the Statistics Department of the University Carlos III de Madrid. Harry Vander Elst gratefully acknowledges financial support from a FRESH grant and David Veredas from the the Czech Science Foundation under project No. 13-32263S. We are also grateful to the conference participants at CFE13 (London, December 2013) and seminars participants at CREATES. Any error and inaccuracy are ours. 


\section{Appendix: supplementary Monte Carlo results}

Table 7: Monte Carlo study - Synchronous trading no noise - results for IQR

\begin{tabular}{ccccccccc}
\hline \hline & \multicolumn{2}{c}{ Model 1 } & \multicolumn{2}{c}{ Model 2 } & \multicolumn{2}{c}{ Model 3} & \multicolumn{2}{c}{ Model 4} \\
& no jumps jumps & no jumps jumps & no jumps jumps & no jumps jumps \\
\hline 30-second returns & & & & & & & & \\
IQR-Ken & 0.109 & 0.1163 & 0.982 & 1.0393 & 0.0869 & 0.0922 & 0.0937 & 0.0999 \\
IQR-Spear & 0.1089 & 0.117 & 0.9812 & 1.0377 & 0.0869 & 0.0917 & 0.0936 & 0.0991 \\
IQR-Quad & 0.1349 & 0.1398 & 0.9822 & 1.0392 & 0.0903 & 0.0951 & 0.1035 & 0.1074 \\
IQR-Gauss & 0.106 & 0.136 & 0.9797 & 1.0377 & 0.0856 & 0.0902 & 0.0915 & 0.0982 \\
\hline 1-minute returns & & & & & & & & \\
IQR-Ken & 0.1598 & 0.1791 & 1.0299 & 1.1177 & 0.1269 & 0.1451 & 0.1365 & 0.1558 \\
IQR-Spear & 0.1589 & 0.1806 & 1.0282 & 1.1143 & 0.1265 & 0.1436 & 0.136 & 0.1535 \\
IQR-Quad & 0.1943 & 0.2082 & 1.0315 & 1.1156 & 0.1307 & 0.1484 & 0.1493 & 0.1659 \\
IQR-Gauss & 0.1544 & 0.211 & 1.0254 & 1.1137 & 0.1245 & 0.1411 & 0.133 & 0.1523 \\
\hline 5-minute returns & & & & & & & & \\
IQR-Ken & 0.4638 & 1.3962 & 1.3923 & 1.9616 & 0.3736 & 0.597 & 0.3956 & 0.6597 \\
IQR-Spear & 0.4512 & 1.3938 & 1.3823 & 1.9362 & 0.3674 & 0.5848 & 0.3872 & 0.6406 \\
IQR-Quad & 0.5217 & 1.3892 & 1.3905 & 1.9412 & 0.3735 & 0.59 & 0.4112 & 0.6503 \\
IQR-Gauss & 0.4399 & 1.4264 & 1.3742 & 1.9301 & 0.3619 & 0.5782 & 0.3808 & 0.6343 \\
\hline 15-minute returns & & & & & & & & \\
IQR-Ken & 0.6592 & 2.5402 & 1.4241 & 3.0661 & 0.5033 & 0.9738 & 0.5385 & 1.1775 \\
IQR-Spear & 0.6483 & 2.5177 & 1.4176 & 3.0217 & 0.501 & 0.9614 & 0.5338 & 1.152 \\
IQR-Quad & 0.7714 & 2.3952 & 1.4551 & 3.0383 & 0.5176 & 0.986 & 0.5828 & 1.1927 \\
IQR-Gauss & 0.6341 & 2.6534 & 1.4063 & 3.0236 & 0.4945 & 0.9525 & 0.5233 & 1.1477 \\
\hline \hline
\end{tabular}

Monte Carlo estimation results for 4 quantile-based volatility estimators of the integrated covariance matrix under four different models specified with and without jumps. The columns report the root mean square errors (RMSE) as computed in (17) for 4000 draws of 23400 observations each corresponding to a situation of one day of 6.5 hours of trading and prices recorded simultaneously each seconds. 


\section{References}

Aït-Sahalia, Y., J. Fan and D. Xiu (2010), "High-Frequency Covariance Estimates with Noisy and Asynchronous Data", Journal of the American Statistical Association, 105, 1504-1517.

Andersen, T., D. Dobrev and E. Schaumburg (2012), "Jump-robust volatility estimation using nearest neighbor truncation", Journal of Econometrics, 169, 74-98.

Andersen, T. G. and T. Bollerslev (1998), "Answering the skeptics: Yes, standard volatility models do provide accurate forecasts", International Economic Review, 39, 885905.

Andersen, T. G., T. Bollerslev, F. Diebold and P. Labys (2001), "The distribution of realized exchange rate volatility", Journal of the American statistical association, 96, 42-55.

(2003), "Modeling and forecasting realized volatility", Econometrica, 71(2), 579-625.

Andreou, E. and E. Ghysels (2002), "Rolling-Sample Volatility Estimators: Some New Theoretical, Simulation and Empirical Results", Journal of Business and Economic Statistics, 20(3), 363376.

Bandi, F. M. and J. R. Russell (2008), "Microstructure Noise, Realized Variance, and Optimal Sampling", Review of Economic Studies, 75 (2), 339-369.

Bannouh, K., D. van Dijk and M. Martens (2009), "Range-based covariance estimation using high-frequency data: the realized co-range", Journal of Financial Econometrics, 7, 341372.

Barndorff-Nielsen, O. E., P. R. Hansen, A. Lunde and N. Shephard (2008), "Designing Realized Kernels to Measure the ex post Variation of Equity Prices in the Presence of Noise", Econometrica, 76(6), 1481-1536.

- (2009), "Realized kernels in practice: Trades and quotes", The Econometrics Journal, 12(3), C1-C32.

(2011), "Multivariate realised kernels: Consistent positive semi-definite estimators of the covariation of equity prices with noise and non-synchronous trading", Journal of Econometrics, 162(2), 149-169.

Barndorff-Nielsen, O. E. and N. Shephard (2004a), "Measuring the impact of jumps in multivariate price processes using bipower covariation", Discussion Paper, Nuffield College, Oxford University.

(2004b), "Power and bipower variation with stochastic volatility and jumps", Journal of financial econometrics, 2(1), 1-37.

(2007), "Variation, jumps, market frictions and high frequency data in financial econometrics", in "Advances in Economics and Econometrics. Theory and Applications, Ninth World Congress", t. 3, p. 328-372.

Bauwens, L., S. Laurent and J. V. Rombouts (2006), "Multivariate GARCH models: a survey", Journal of applied econometrics, 21(1), 79-109.

Bollerslev, T. (1986), "Generalized autoregressive conditional heteroskedasticity", Journal of econometrics, 31(3), $307-327$.

(1990), "Modelling the coherence in short-run nominal exchange rates: a multivariate generalized ARCH model", The Review of Economics and Statistics, 72, 498-505.

Boudt, K., J. Cornelissen and C. Croux (2011a), "The Gaussian rank correlation estimator: robustness properties", Statistics and Computing, 22, 471-483. 
(2012), "Jump robust daily covariance estimation by disentangling variance and correlation components", Computational Statistics and Data Analysis, 56(11), 2993-3005.

Boudt, K., C. Croux and S. Laurent (2011b), "Outlyingness Weighted Covariation", Journal of Financial Econometrics, 9(4), 657-684.

Boudt, K. and J. Zhang (2013), "Jump robust two time scale covariance estimation and realized volatility budgets", Quantitative Finance, (ahead-of-print), 1-14.

Carr, P., H. Geman, D. B. Madan and M. Yor (2002), "The Fine Structure of Asset Returns: An Empirical Investigation", Journal of Business, 75(2), 305-332.

Christensen, K., S. Kinnebrock and M. Podolskij (2010a), "Pre-averaging estimators of the ex-post covariance matrix in noisy diffusion models with non-synchronous data", Journal of Econometrics, 159(1), 116-133.

Christensen, K., R. Oomen and M. Podolskij (2010b), "Realised quantile-based estimation of the integrated variance", Journal of Econometrics, 159, 74-98.

Christensen, K., M. Podolskij and M. Vetter (2013), "On covariation estimation for multivariate continuous Itô semimartingales with noise in non-synchronous observation schemes", Journal of Multivariate Analysis, 120 , 59-84.

Corsi, F., S. Peluso and F. Audrino (2012), "Missing in asynchronicity: A Kalman-EM approach for multivariate realized covariance estimation", Discussion Paper, Department of Economics University of St. Gallen.

Croux, C. and C. Dehon (2010), "Inuence functions of the Spearman and Kendall correlation measures", Statistical Methods \& Applications, 19(4), 497-515.

Dacorogna, M., R. Gencay, U. Muller, R. Olsen and O. Pictet (2001), An Introduction to High Frequency Finance, Academic Press.

De Pooter, M., M. Martens and D. Van Dijk (2008), "Predicting the daily covariance matrix for S\&P 100 stocks using intraday data but which frequency to use?", Econometric Reviews, 27, 19922.

Engle, R. (2002), "Dynamic conditional correlation: A simple class of multivariate generalized autoregressive conditional heteroskedasticity models", Journal of Business E Economic Statistics, 20(3), 339-350.

Engle, R. and K. Kroner (1995), "Multivariate simultaneous generalized ARCH", Econometric theory, 11(01), $122-150$.

Engle, R. F. (1982), “Autoregressive conditional heteroscedasticity with estimates of the variance of United Kingdom inflation", Econometrica, 159, 987-1007.

Epps, T. W. (1979), "Comovements in stock prices in the very short run", Journal of the American Statistical Association, 74(366a), 291-298.

Falk, M. (1998), "A note on the comedian for elliptical distributions", Journal of Multivariate Analysis, 67(2), $306-317$.

Fan, J., Y. Li and K. Yu (2012), "Vast Volatility Matrix Estimation Using High-Frequency Data for Portfolio Selection", Journal of the American Statistical Association, 107(497), 412-428. 
Fleming, J., C. Kirby and B. Ostdiek (2001), "The economic value of volatility timing", The Journal of Finance, $56(1), 329-352$.

(2003), "The economic value of volatility timing using realized volatility", Journal of Financial Economics, 67,473509 .

Foster, D. P. and D. B. Nelson (1996), "Continuous Record Asymptotics for Rolling Sample Variance Estimators", Econometrica, 64(3), 139174

Halbleib, R. and V. Voev (2011), "Forecasting Covariance Matrices: A Mixed Frequency Approach", CREATES Research Papers - 2011-03.

Harris, F., T. McInish, G. Shoesmith and R. Wood (1995), "Cointegration, error correction and price discovery on informationally-linked security markets", Journal of Financial and Quantitative Analysis, 30, 563-581.

Hautsch, N., L. M. Kyj and R. Oomen (2012), "A blocking and regularization approach to high-dimensional realized covariance estimation", Journal of Applied Econometrics, 27(4), 625-645.

Hayashi, T. and N. Yoshida (2005), "On covariance estimation of non-synchronously observed diffusion processes", Bernoulli, 11(2), 359-379.

Jacod, J., Y. Li, P. Mykland, M. Podolskij and M. Vetter (2009), "Microstructure noise in the continuous case: the pre-averaging approach", Stochastic Processes and Their Applications, 119, 2249-2276.

Jacod, J. and V. Todorov (2009), "Testing for common arrivals of jumps for discretely observed multidimensional processes", The Annals of Statistics, 1792-1838.

Kendall, M. (1938), "A new measure of rank correlation", Biometrika, 30, 81-93.

Mancini, C. and F. Gobbi (2012), "Identifying the Brownian covariation from the co-jumps given discrete observations", Econometric Theory, 28(2), 249-273.

Martens, M. (2004), "Estimating unbiased and precise realized covariances", Department of Finance, Erasmus School of Economics, Rotterdam.

McAleer, M. and M. Medeiros (2008), "Realized volatility: a review", Econometric Reviews, 27, 10-45.

Mosteller, F. (1946), "On Some Useful "Inefficient" Statistics", Annals of Mathematical Statistics, 17(4), 377-408.

Mykland, P. A. and L. Zhang (2009), "Inference for continuous semimartingales observed at high frequency", Econometrica, 77(5), 1403-1445.

Noureldin, D., N. Shephard and K. Sheppard (2012), "Multivariate high-frequency-based volatility (HEAVY) models", Journal of Applied Econometrics, 27(6), 907-933.

Pasman, V. and G. Shevlyakov (1987), "Robust methods of estimating the correlation coefficient", Avtomatika $i$ Telemekhanika, (3), 70-80.

Podolskij, M. and M. Vetter (2009), "Estimation of volatility functionals in the simultaneous presence of microstructure noise and jumps", Bernoulli, 15(3), 634-658.

Protter, P. E. (2004), Stochastic Integration and Differential Equations: Version 2.1, t. 21, Springer. 
Rousseeuw, P. J. and C. Croux (1993), "Alternatives to the median absolute deviation", Journal of the American Statistical Association, 88, 1273-1283.

Shephard, N. and D. Xiu (2012), "Econometric analysis of multivariate realised QML: estimation of the covariation of equity prices under asynchronous trading", Tech. rep., University of Oxford and University of Chicago.

Shevlyakov, G. and P. Smirnov (2011), "Robust Estimation of the Correlation Coefcient: An Attempt of Survey", Austrian Journal of Statistics, 40(1\&2), 147-156.

Spearman, C. (1904), "General intelligence objectively determined and measured", The American Journal of Psychology, 15(2), 201-292.

Tse, Y. K. and A. Tsui (2000), "A multivariate GARCH model with time-varying correlations", Available at SSRN 250228, 20, 351-362.

Voev, V. and A. Lunde (2007), "Integrated Covariance Estimation using High-frequency Data in the Presence of Noise", Journal of Financial Econometrics, 5(1), 68-104.

Zhang, L. (2006), "Efficient estimation of stochastic volatility using noisy observations: A multi-scale approach", Bernoulli, 12(6), 1019-1043.

(2011), "Estimating Covariation: Epps Effect, Microstructure Noise", Journal of Econometrics, 160, 33-47.

Zhang, L., P. Mykland and Y. Aït-Sahalia (2005), "A tale of two time scales: Determining integrated volatility with noisy high frequency data", Journal of The American Statistical Association, 100(472), 1394-1411.

Zhou, B. (1996), "High-Frequency Data and Volatility in Foreign Exchange Rates", Journal of Business and Economic Statistics, 14, 45-52. 